Florida International University FIU Digital Commons

4-8-1986

\title{
Pulsed parabolic equation model of acoustic transmission (PPEMAT)
}

Alicia Gonzalez Acebo

Florida International University

DOI: $10.25148 /$ etd.FI13101517

Follow this and additional works at: https://digitalcommons.fiu.edu/etd

Part of the Computer Sciences Commons

\section{Recommended Citation}

Acebo, Alicia Gonzalez, "Pulsed parabolic equation model of acoustic transmission (PPEMAT)" (1986). FIU Electronic Theses and Dissertations. 1084.

https://digitalcommons.fiu.edu/etd/1084

This work is brought to you for free and open access by the University Graduate School at FIU Digital Commons. It has been accepted for inclusion in FIU Electronic Theses and Dissertations by an authorized administrator of FIU Digital Commons. For more information, please contact dcc@fiu.edu. 


\begin{abstract}
PULSED PARABOLIC EQUATION MODEL OF ACOUSTIC TRANSMISSION (PPEMAT)
\end{abstract}

by

Alicia González Acebo

Underwater sound is very important in the field of oceanography where it is used for remote sensing in much the same way that radar is used in atmospheric studies. One way to mathematically model sound propagation in the ocean is by using the parabolic-equation method, a technique that allows range dependent environmental parameters. More importantly, this method can model sound transmission where the source emits either a pure tone or a short pulse of sound. Based on the parabolic approximation method and using the split-step Fourier algorithm, a computer model for underwater sound propagation was designed and implemented. This computer model differs from previous models in its use of the interactive mode, structured programing, modular design, and state-of-the-art graphics displays. In addition, the model maximizes the efficiency of computer time through synchronization of loosely coupled dual processors and the design of a restart capability. Since the model is designed for adaptability and for users with limited computer ski11s, it is anticipated that it will have many applications in the scientific community. 
PULSED PARABOLIC EQUATION MODEL OF ACOUSTIC TRANSMISSION (PPEMAT)

by

Alicia González Acebo

A thesis submitted in partial fulfillment of the requirements for the degree of

MASTER OF SCIENCE

in

COMPUTER SCIENCE

at

FLORIDA INTERNATIONAL UNIVERSITY

Committee in charge:

Professor Robert C. Fisher, Chairperson

Professor Toby Berk

Professor John C. Comfort

Professor Linda M. Laws on

Apri1 1986 
To Professors: Robert C. Fisher

Toby Berk

John C. Comfort

LInda M. Lawson

This thesis, having been approved in respect to form and mechanical execution, is referred to you for judgment upon its substantial merit.

The thesis of Alicia González Acebo is approved.

Dean James A. Mau

College of Arts and Sciences

Professor Linda M. Laws on

Professor Toby Berk

Professor Johr C. Coxfort

Pfofessor Robert C". Fisher

Date of Examination: 
PULSED PARABOLIC EQUATION MODEL OF ACOUSTIC TRANSMISSION (PPEMAT)

by

Alicia González Acebo

A thesis submitted in partial fulfillment of the requirements for the degree of

MASTER OF SCIENCE

in

COMPUTER SCIENCE

at

FLORIDA INTERNATIONAL UNIVERSITY

1986 


\section{ACKNOWLEDGMENTS}

I would like to thank my thesis director Linda M. Lawson for all her help and for making this thesis possible.

I'm also grateful to the National Oceanic and Atmospheric Administration and its personnel, especially David Palmer, Gall Derr, Yeun-Ho Daneshzadeh, Thomas Fleming, William Nodal and David Senn, for the assistance and facilities necessary to develop this project.

I would like to thank my parents for their continued support and encouragement now and always.

Special thanks go to Lynn and Toby Berk, and Robert C. Fisher for their help and encouragement.

Last, and most important, I express my appreciation to my children, Lici, Alexandra and Daniel, for motivating me and for doing all the housework during the past two years. 
LIST OF TABLES............................ v

LIST OF FIGURES............................ vi

I. InTRODUCTION................................ 1

II . MODEL DEVELOPMENT............................. 4

III. MATHEMATICAL DESCRIPTION........................ 9

IV. MODEL DESCRIPTION............................. 16

A. Overview................................. 16

B. Command Language Description.................... 26

C. Source Language Description..................... 40

V. USER'S GUIDE............................... 58

A. How to Run the Mode1....................... 58

B. File structure............................ 64

C. Utility Programs........................... 76 
TABLE OF CONTENTS (continued)

Vi. SAMPle RUnS $\ldots \ldots \ldots \ldots \ldots \ldots \ldots \ldots \ldots \ldots \ldots \ldots \ldots \ldots \ldots \ldots, 77$

VII. FUTURE MODIFICATIONS.........................92

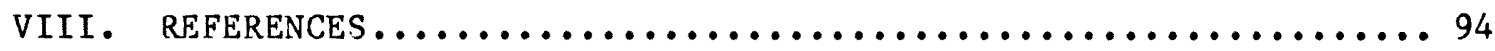

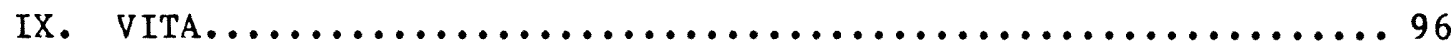

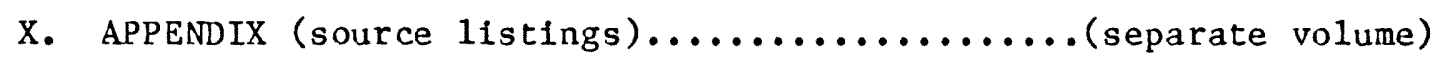


LIST OF TABLES

Table 1. File Description, CW Model.....................67

Table 2. File Description, CW Model (continued)..............68

Table 3. File Description, Pulsed Mode1...................69

Table 4. File Description, Pulsed Mode1 (continued)...........70 


\section{LIST OF FIGURES}

Figure 1. Outline of Model structure (CW Version)............17

Figure 2. Outline of Mode1 Structure (PSP CW Module).......... 18

Figure 3. Outline of Model Structure (PSP Pulse Module)........ 19

Figure 4. Source Code Structure for $\mathrm{CW}$ and PSP CW (PLSCW)....... 19

Figure 5. Initializing Routines for $C W$ Version............... 20

Figure 6. Calculation Routines for CW Version............... 21

Figure 7. Schematic of Contour Plots for $C W$ Version............ 22

Figure 8 . Schematic of Line Plots for $\mathrm{CW}$ Version............. 22

Figure 9. Initializing Routines for PSP CW Module............ 23

Figure 10. Calculation Routines for PSP CW Module............. 24

Figure 11. Schematic of Routines in PSP Pulse Module........... 25

Figure 12. Transmission Loss Contours - Parabolic Profile (Uniras).................................. 78

Figure 13. Transmission Loss Contours - Parabolic Profile (IGL).... 79

Figure 14. Transmission Loss Contours - Source Depth 130 Meters.... 80 
LIST OF FIGURES (continued)

Figure 15. Transmission Loss Contours - Source Depth 1200

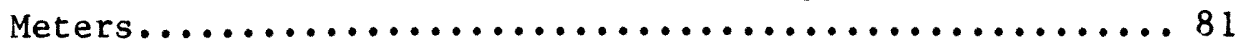

Figure 16. Transmission Loss Contours - Source Depth 5200 Meters................................. 82

Figure 17. Transmission Loss Contours - Range 500 Kilometers...... 84

Figure 18. Line P1ot - Depth 130 Meters, Range 500 Kilometers..... 85

Figure 19. Pulsed Plot - Depth 3000 Meters................. 86

Figure 20. Pulsed P1ot - Depth 1000 Meters................. 87

Figure 21. Pulsed Plot - Depth 6000 Meters..................88

Figure 22. Pulsed Plot - Depth 3000 Meters (Default Plot Specifications)........................... 89

Figure 23. Pulsed P1ot - Depth 1000 Meters (Default Plot Specifications)........................... 90

Figure 24. Pulsed Plot - Depth 6000 Meters (Default P1ot

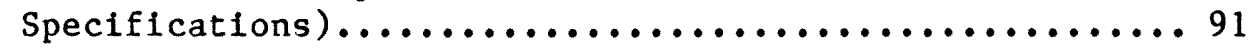




\section{INTRODUCTION}

Sound is one of the best tools oceanographers have for working in the sea. If offers them the ability to listen to underwater life, measure distances, map the bottom terrain, navigate, track submarines and ships, search for ofl and mineral deposits, and generally explore the ocean environment [14]. The reason for its wide spread use becomes clear if one compares the properties of the atmosphere with those of the ocean. We know a lot about the atmosphere because we observe it using visible light and radar. Unfortunately, the ocean is opaque to this type of radiation. We do not see much by looking down into the ocean nor can we probe the ocean using radar. The ocean is, however, transparent to sound. A classic experiment was conducted in 1960 which illustrates this point. The sounds from depth charges fired by a ship off Australia were picked up some $33 / 4$ hours later near Bermuda at a distance of 19,000 kilometers, or half-way around the world. Because underwater sound travels so well it plays the role in investigations of the ocean that visual sightings and radar play in investigations of the atmosphere [14].

All the applications of underwater sound can be described in the same general way. There is a naturally occurring or man-made source of sound. The sound from this source travels through the water, encountering changing conditions or objects along the way, and is received by an underwater microphone called a hydrophone. From an analysis of the signal recelved by the hydrophone inferences are made about the source or about the conditions or objects encountered along the transmission path. It is clearly important that there be a good physical understanding of the transmission process. To a large degree this under- 
standing has come from numerical simulations using a digital computer and a more comprehensive and accurate approximation to equations describing sound propagation (called the parabolic equation method [18]).

Many sound transmission models have been developed based on the parabolic-equation method since its introduction to underwater acoustics. However, almost all of these models were developed by modifying a few early models [4], [8], [11]. The computers for which these early models were designed had limited accessible memory and barely resemble the machines available today. Although the more recent models differ in many ways, they were developed before any serious studies on structured programing and design were available and, because of the evolutionary manner in which they were developed, they are very difficult to analyze and in some cases were never completely debugged. One of the difficulties in modelling acoustic transmission at long ranges is that changes can occur along the transmission path in the speed of sound. Although progress has been made in increasing flexibility, there remained the need for a model that will permit a variety of range-dependent parameters as input. More importantly, there was a distinct need for a program that can be used for multiple frequencies rather than a single frequency so that a pulsed source could be mode1led.

The use of advanced technology and programming methods permitted the design and implementation of a distinctly improved model described in this thesis. This model is a computational-bound type mode1. It confronts space problems because of the large amounts of data that are handled, and time problems due to the number of hours taken by the 
computation, 1.e., 6 to 37 hours. The space problem was solved by the virtual memory facility offered by modern computers and a good, flexible design of the input/output structure of the results. The time problem was solved by using the operating system command language to synchronize the two loosely coupled processors that were avallable and to implement a restart mechanism in case of system failure. The range-dependent changes that traditionally have been difficult to model were all considered in the design of the one presented in this thesis.

This model offers a very easy-to-use package. It runs in an interactive mode and is self-directed. The user needs to know only one or two commands to execute the command language driver programs that create files, execute the model, submit synchronized batch jobs and/or plot different kinds of graphics.

Because of the model design and ease with which it can be run, it is thought that the model will have many applications ranging from investigations of the grunts emitted by whales to studies of the sound resulting from marine earthquakes.

The thesis is organized as follows. Section II contains a description of the model development. Section III gives a brief description of the equations for the parabolic approximation and the split step algorithm. The model structure is described in Section IV with a user's guide in Section $V$. Various examples of outputs and plots are provided in Section VI. Suggestions for future modifications that might prove useful are given in Section VII. References are given in Section VIII, a vita is provided in Section IX, and listings of the code are provided in the Appendix under separate cover. 


\section{MODEL DEVELOPMENT}

A computer program was developed based on the parabolic-equation method which can be used to model underwater sound transmission at long range and in those special cases in which the source emits a short pulse of sound (long range means distances orders of magnitude larger than the water depth, i.e., hundreds or even thousands of kilometers).

Developing a computer model which would meet these requirements necessitated background reading and research in the parabolic equation method and necessary mathematical techniques such as the Fast Fourier transform (FFT) [1], [3], [6], [7], [10], [12]. It was also necessary to learn the basic characteristics of sound transmission in the ocean [5], [13]. Learning about the Atlantic Oceanographic and Meteorological Laboratory's (AOML) computer facilities required golng through instruction computer programs, reading manuals, talking to other programmers, and running some tests on the FFT's routines.

The model consists of two different versions. The simplest version calculates and displays the level of sound in a region of the ocean produced by a single frequency, or continuous wave (CW) source. The more complex version calculates the pressure as a function of time for specified points using a short pulse of sound for the source. The first version will be called the $C W$ version and the later the pulse sound propagation (PSP) version.

The computer facilities consisted primarily of two loosely coupled VAX $11 / 785$ processors. The model was a computational-bound computer problem, which required a variety of different input data. Because of the extremely large number of calculations required for the PSP version, and hence long CPU times, it was necessary to develop a method for 
dividing the computations between the two processors. This required the design of a method of synchronizing the two processors. Moreover, in view of the long CPU times, a system was included to restart the calculations in the event of a system fallure.

The primary goal of the design was to produce a program that could be run by entering a single word. In order to accomplish this goal it was necessary to use extensively the VAX system command language which resembles a high level programming language. The command language programs formed the underlying structure for a complex system that performs a variety of tasks. These tasks include jobs such as creating input files, submitting batch jobs, synchronizing the two processors, executing programs and/or plotting different kinds of graphics.

Fortran was used as the programming language for the mathematical computations because of its widespread use in the scientific community. Once the algorithms were designed it was necessary to develop the structure of the Fortran modules. Although top-down design is widely recognized as the best approach, it only works if the problem can be completely envisioned from the start. Because of the difficulties involved in visualizing the model as a whole, the $\mathrm{CW}$ design was done first. Even visualizing the interaction of the CW model's parts was problematic, since there were at least three factors at work: range, depth and frequency. Therefore, small structured routines were designed to do the calculations which were later fit together. Hence the complexity of this problem made a top-down approach very difficult and thus a bottom-up approach was used in the initial development of the CW version. 
Once the design for the $\mathrm{CW}$ was concelved, it was possible to visualize the design of the PSP according to a top-down structure. Primarily because of major differences in input/output and run times required, the $\mathrm{PSP}$ version was not only written completely independent of the CW version, but was also divided into two parts. The first part, called the PSP CW roodule, resembles the calculations for the CW version with an additional loop over frequencies. Because of its similarity to the CW version, the PSP CW module invokes many of the same subroutines for the executable calculations. The second module uses the information from PSP CW and a description of the source signal to produce a time history of the real pressures and phases at the selected points. This module is called the PSP Pulse module.

In order to efficiently display many numbers the graphics facilities were considered in the model design. These facilities depend primarily on the Tektronix 4107 computer display terminal, hence the model was designed with this terminal in mind. The 4107 is a microprocessor-controlled terminal, designed specially for text editing and color graphics display. The avallable printers were Tektronix 4695 color graphics copiers. These coplers are low-cost, seven-color printers that use three colors of ink plus black ink to produce crisp, multicolor text, line drawings, and graphics. These coplers use ink-jet technology to print anything that can be displayed on the Tektronix color graphics terminals. Plotting routines were developed, using a top down design, to plot model results and to use for debugging separate modules. One such set of routines is a line-plotting package that uses the Tektronix 4010C01 Plot 10 Interactive Graphics Library (IGL) routines. This line-plotting package is called by both the CW and the 
PSP versions of the model. Two pre-existing contour packages, used only by the $C W$ version, were incorporated in the model. One coutour package, again IGL routines, produces a color line contour. The second type of contour uses Uniras routines. The Uniras package is built on top of Raspak, a specially designed raster graphics software tool box for the application programmer. Special drivers were designed for the plot packages.

In all of the plots the usefulness of natural associations were incorporated in the design. As an example, consider the use of transmission loss as a measure of the level of sound at a given location. Transmission loss is given in decibels $(d B)$ and is a relative measure of how much sound is lost during propagation from one point to another. Since high transmission loss values represent regions of less sound (i.e., quieter regions), it is customary to invert the transmission loss values so that peaks correspond to loud regions and valleys to quieter areas. Therefore, it was necessary to have a plot package with the flexibility to flip axes and values. The thoroughly debugged and easyto-use line plot graphics package designed allowed data and/or axes to be flipped in any direction; it also normalizes the data values if necessary. A11 the plots in both versions are run interactively letting the user select the format at the time the plotting is being done.

The "one-word" design characteristic of the model allows the user to run the model and create plots as easily as possible. This special easy-to-use, self-directed package was designed for a non-technical user. The "package," which uses the digital command language (DCL), prompts the user for all information, such as values for range, depth, and frequency variables. It also prompts for plot specifications and for the choice of graphics to plot. 
The complex features of the model such as the synchronization of the two processors, the restart mechanism, the creation, execution and deletion of temporary command procedure files are all user-transparent. The model possesses several other features. The programs are well documented and all the parameters to dimension arrays are in one module that is accessed by every program that needs it. The model runs in a scratch workspace when temporary files are created. It creates these files with unique names, that are deleted after they are used. This is all user-transparent and the process returns to the original user node when it finishes. A file naming scheme was developed to help the user keep track of the files. This scheme is suggested in the prompts, but it is not obligatory. 
CW Transmission

The vibrating part of the water pressure which a hydrophone measures is called the sound pressure. It depends on both time and on location and is determined by solving the wave equation

$$
\left(\nabla^{2}-\frac{1}{c^{2}} \frac{\partial^{2}}{\partial t^{2}}\right) p(x, y, z, t)=0
$$

where

$$
\nabla^{2} \equiv \frac{\partial^{2}}{\partial x^{2}}+\frac{\partial^{2}}{\partial y^{2}}+\frac{\partial^{2}}{\partial z^{2}}
$$

The speed of sound $c$ depends on position, $c=c(x, y, z)$.

Since the formalism which follows depends heavily on what is called frequency it is worthwhile to briefly discuss the concept. The number of vibrations of the seawater in a second is called the frequency of the sound. For example, a frequency of 200 cycles per second means that small portions of the fluid are oscillating back and forth 200 times each second. The unit of frequency is call the Hertz (Hz) so that a frequency of $200 \mathrm{~Hz}$ is the same as a frequency of 200 cycles per second. (As a point of reference, musical instruments are tuned according to frequency. The frequency determines the pitch of a tone; the greater the frequency, the higher the pitch. The standard in music is that the first A above middle $\mathrm{C}$ has a frequency of $440 \mathrm{~Hz}$. )

If the sound emitted by a source consists of a pure tone, 1.e., a single frequency $f$, so that close to the source the time dependence of the pressure can be written $\cos (2 \pi f t)$, then the sound pressure far from the source will also consist of a pure tone and can be expressed as the real part of the quantity 


$$
P(x, y, z) \exp (-2 \pi i f t)
$$

where $P(x, y, z)$ satisfies the Helmholtz equation

$$
\left(\nabla^{2}+\left(\frac{2 \pi f}{c}\right)^{2}\right) P(x, y, z)=0
$$

It is convenient to normalize the sound speed by some arbitrary reference sound speed $c_{0}$ so that the Helmholtz equation becomes

$$
\left(\nabla^{2}+k_{0}^{2} n^{2}\right) P=0
$$

where $n=c_{0} / c(x, y, z)$ is called the index of refraction and $k_{0}=2 \pi f / c_{0}$ is called the characteristic wave number.

Sound transmission such as this, described by a single frequency, is called continuous wave or CW transmission.

\section{Parabolic-Equation Method}

The parabolic-equation method, developed by Tappert and Hardin [9], [16], [17], [18] consists of two parts; the parabolic approximation and numerical implementation using the split-step Fourier algorithm.

In the following the coordinate system will be orientated so that the z-axis is positive downward from the surface of the ocean. The horizontal coordinates are then $x$ and $y$. The horizontal distance between the source and the observation point is

$$
r=\sqrt{x^{2}+y^{2}}
$$

The parabolic approximation assumes that the solution to the Helmholtz equation, Eq. (5), for the pressure is not so much different 
from the solution to a simpler equation called the parabolic equation. One assumes

$$
P \approx \frac{e^{1 k} o^{r}}{\sqrt{r}} \psi(r, z)
$$

where $\psi$ obeys the parabolic equation

$$
\left(2 i k_{0} \frac{\partial}{\partial r}+\frac{\partial^{2}}{\partial z^{2}}+k_{0}^{2}\left(n^{2}(z, r)-1\right)\right) \psi=0
$$

and

$$
n(r, z)=c_{0} / c(r, z)
$$

where $c(r, z)$ is the sound speed in the horizontal plane containing the sound source and the recelving hydrophone. The solution to Eq. (8) satisfies the same boundary conditions at the top and bottom of the ocean as $\mathrm{P}$ satisfies and an initial condition. This initial condition is usually specified by requiring that $\psi$ equal some depth-dependent function as a small horizonal distance $\varepsilon$ from the source. That is

$$
\psi(\varepsilon, z)=h(z)
$$

The precise form of $h$ and the value of $\varepsilon$ depend on the properties of the source and the acoustic properties of the ocean in the region of the source.

The split-step Fourier algorithm is a very fast and efficient numerical algorithm for solving Eq. (8). The field $\psi$ is used to calculate the fleld at a somewhat greater range. This new field is then used to calculate the field at an even greater range. The process is 
continued until the field is known at all ranges. The particular form for the split-step Fourier algorithm depends on the boundary conditions satisfied at the top and bottom of the ocean. For the case where the medium is unbounded, the algorithm reads

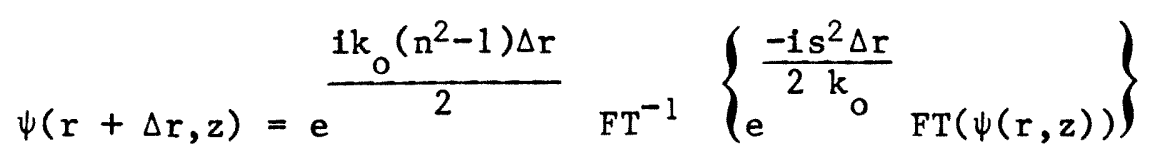

where FT is the Fourier transform

$$
\operatorname{FT}(\psi(r, z))=\int_{-\infty}^{\infty} d z e^{i s z} \psi(r, z)
$$

and $\mathrm{FT}^{-1}$ is the corresponding inverse Fourier transform.

Since it is necessary to work with discrete functions in a bounded medium, the above equations must be re-cast in a discrete and finite form. Let $\psi(r, z)$ be sampled in depth so that we will use the following definition

$$
\psi\left(r, z_{k}\right)=\left\{\begin{array}{l}
\psi(r, k \Delta z), 0 \leq k \Delta z \leq z_{\max } \text { for } 0 \leq k \leq N-1 \\
0, \text { otherwise. }
\end{array}\right.
$$

The length of the depth interval defines the fundamental wavenumber, $1 / z_{\max }=\eta_{0} \cdot$ The Fourier transform of $\psi, \Psi$, is sampled at multiples of $n_{0}$, i.e.,

$$
\Psi\left(\mathrm{n} \eta_{0}\right)=\Psi\left(\mathrm{n} / \mathrm{z}_{\max }\right)=\Psi(\mathrm{n} / \mathrm{N} \Delta \mathrm{z}), 0 \leq \mathrm{n} \leq \mathrm{N}-1
$$

where the original function was sampled at $\mathrm{N}$ points. By defining 


$$
\alpha_{n}=\sum_{k=0}^{N-1} \psi(r, k \Delta z) e^{-2 \pi i(k \Delta z)\left(n n_{0}\right)}=\sum_{k=0}^{N-1} \psi(r, k \Delta z) e^{-2 \pi i k n / N}
$$

one can show that

$$
\psi(r, k \Delta z)=\frac{1}{N} \sum_{n=0}^{N-1} \alpha_{n} e^{2 \pi i k n / N}
$$

By defining $\alpha_{n}=\Psi(n / N \Delta z)$ we have the discrete Fourier transform equations

$\psi(r, k \Delta z)=\frac{1}{N} \sum_{n=0}^{N-1} \Psi(n / N \Delta z) e^{2 \pi i k n / N}$ and $\Psi(n / N \Delta z)=\sum_{k=0}^{N-1} \psi(r, k \Delta z) e^{-2 \pi i k n / N}$

which we will write as

$$
\Psi(n)=\operatorname{FT}\{\psi(r, z)\} \text { and } \psi(r, z)=\operatorname{FT}^{-1}\{\Psi(n)\} \text {. }
$$

One assumes that $\psi$ vanishes at $z=0$, the top of the ocean, and at $z=$ $\mathrm{L}$, the bottom of the ocean. These assumptions allow the transform of $\psi$ to be written as a sine transform

$$
\Psi(n / N \Delta z)=2 \sum_{k=0}^{N-1} \psi(r, k \Delta z) \sin \frac{\pi k n}{N}
$$

Hence, the fact that $\psi$ is odd allows the Fourier transform to be replaced by the sine transform. Also, since the transform of a real, odd function is itself imaginary and odd, one is able to use the same routine to calculate the inverse transform. 
Using these discrete representations, (11) can be written

$$
\psi(r+\Delta r, z)=C_{1} F^{-1}\left\{C_{2} F T[\psi(r, z)]\right\}
$$

where

$$
C_{1}=\exp \left(i k_{0}\left(n^{2}-1\right) \Delta r / 2\right), \text { and } C_{2}=\exp \left(-i s^{2} \Delta r / 2 k_{0}\right) \text {. }
$$

This expression shows that one can calculate the solution at $r+\Delta r$ given the solution at $r$ and thereby allows the entire pressure field to be calculated by marching out in range. The algorithm is very fast because it uses a fast Fourier sine transform (FFT) algorithm [2], [6], [7] to compute the required transforms.

\section{Pulsed Parabolic Equation}

By Fourier's theorem it is known that a pulse of sound is composed of a large number of pure tones. Therefore the sound pressure from a pulsed sound source is the sum the CW sound pressures for a large number of frequencies. Instead of $\mathrm{Eq}$. (1) we must now consider the wave equation

$$
\left\{\nabla^{2}-\frac{1}{c^{2}} \frac{\partial^{2}}{\partial t^{2}}\right\} p(x, y, z, t)=F(x, y, z, t)
$$

where $F(x, y, z, t)$ describes the source function. Since the source is composed of a mixture of frequencies, and is localized in space, it can be written in terms of a Fourier transform as

$$
F(x, y, z, t)=\delta\left(\bar{x}-\bar{x}_{s}\right) \text { Real }\{F T(H(f))\}
$$


where $\bar{x}_{s}$ is the position vector of the source. Recall that for a single frequency we have

$$
P_{f}(r, z)=\exp \left(i k_{o} r\right) / \sqrt{r} \cdot \psi_{f}(r, z)
$$

where $P$ and the envelope function $\psi$ now have subscripts $f$ to emphasize the fact that these depend on frequency. For pulsed propagation, instead of (3) we now can write the sound pressure as

$$
\begin{aligned}
p(r, t) & =\operatorname{Real}\left\{F T\left[H(f) P_{f}(r, z)\right]\right\} \\
& =\operatorname{Real}\left\{F T \frac{e^{i k_{o} r}}{\sqrt{r}} \cdot H(f) \cdot \psi_{f}(r, z)\right\} \\
& =\operatorname{Real} \sum_{k=0}^{N-1} \frac{e^{i k} r}{\sqrt{r}} H\left(f_{k}\right) \cdot \psi_{f_{k}}(r, z) e^{-2 \pi f_{k} t}
\end{aligned}
$$

Here the number of terms in this sum, $N$, the values for the frequencies $f_{1}, f_{2}, \ldots f_{k}$, and the transform $H(f)$ depend on the details of the pulse; different pulse shapes have different values for these parameters. The pulsed model evaluates this expression, where the field $P$ is found using the parabolic approximation for each frequency for all frequencles needed to describe the pulse. The sum over frequency is then evaluated using the FFT algorithm. 


\section{MODEL DESCRIPTION}

A. Overview

This model was written for use on the VAX-11/785 dual processor with virtual memory system (VMS) operating system. The programs were compiled and linked into executable modules that are run from command procedures.

The model consists of two separate parts, the $C W$ version and the Pulsed version. The DCL routines for the $C W$ version are outlined in Figure 1. The Pulsed version is divided into two parts, the PSP CW module and the PSP Pulse module. The DCL structure for these modules is provided in Figures 2 and 3, respectively.

As indicated in Figure 1, there are three executable elements in the CW version: CW.EXE, DRVCON*.EXE, and DRVLPD.EXE. An outline of the routines linked together for the CW executable element is provided in Figures 4,5 , and 6 . Figure 4 indicates a division of the $\mathrm{CW}$ routines into those related to initialization of parameters and those used for computations. Figure 5 shows the structure of the initializing routines, while Figure 6 gives the calculating routines. The other two executable elements are the contour and line plotting programs. The contour executable can be either DRVCONUNI.EXE for Uniras plots, or DRVCONIGL.EXE for IGL plots. The schematic for both of these is provided in Figure 7 . The line plot routines 1inked into DRVLPD.EXE are shown in Figure 8.

The PSP CW module, as outlined in Figure 2 , has one executable module, PLSCW.EXE. Here again the inftialization and computational routines are separated and are described in Figures 4, 9, and 10. One should note the similarities of the structure in PSP CW and CW by 


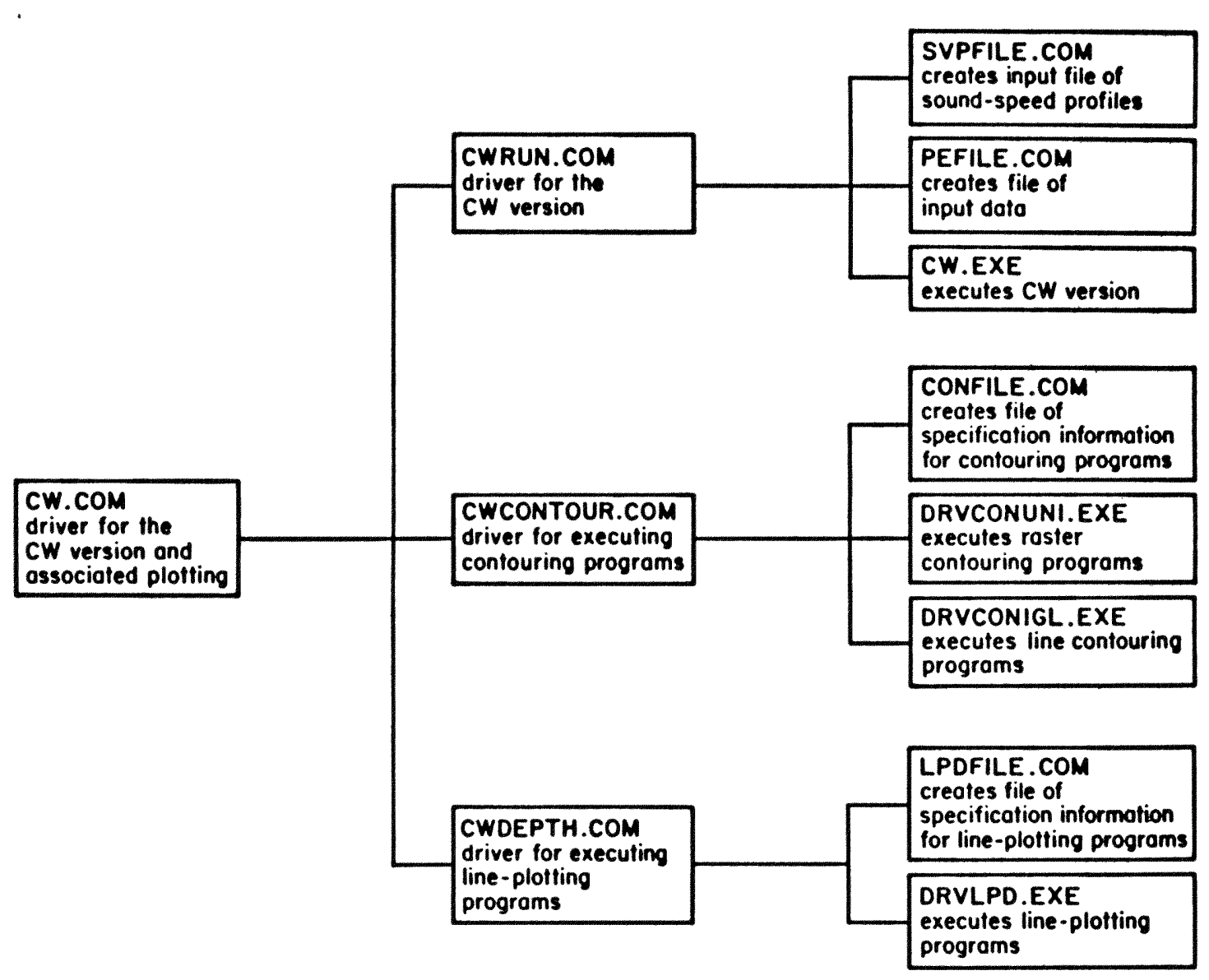

Figure 1. Outline of Model Structure (CW Version). 


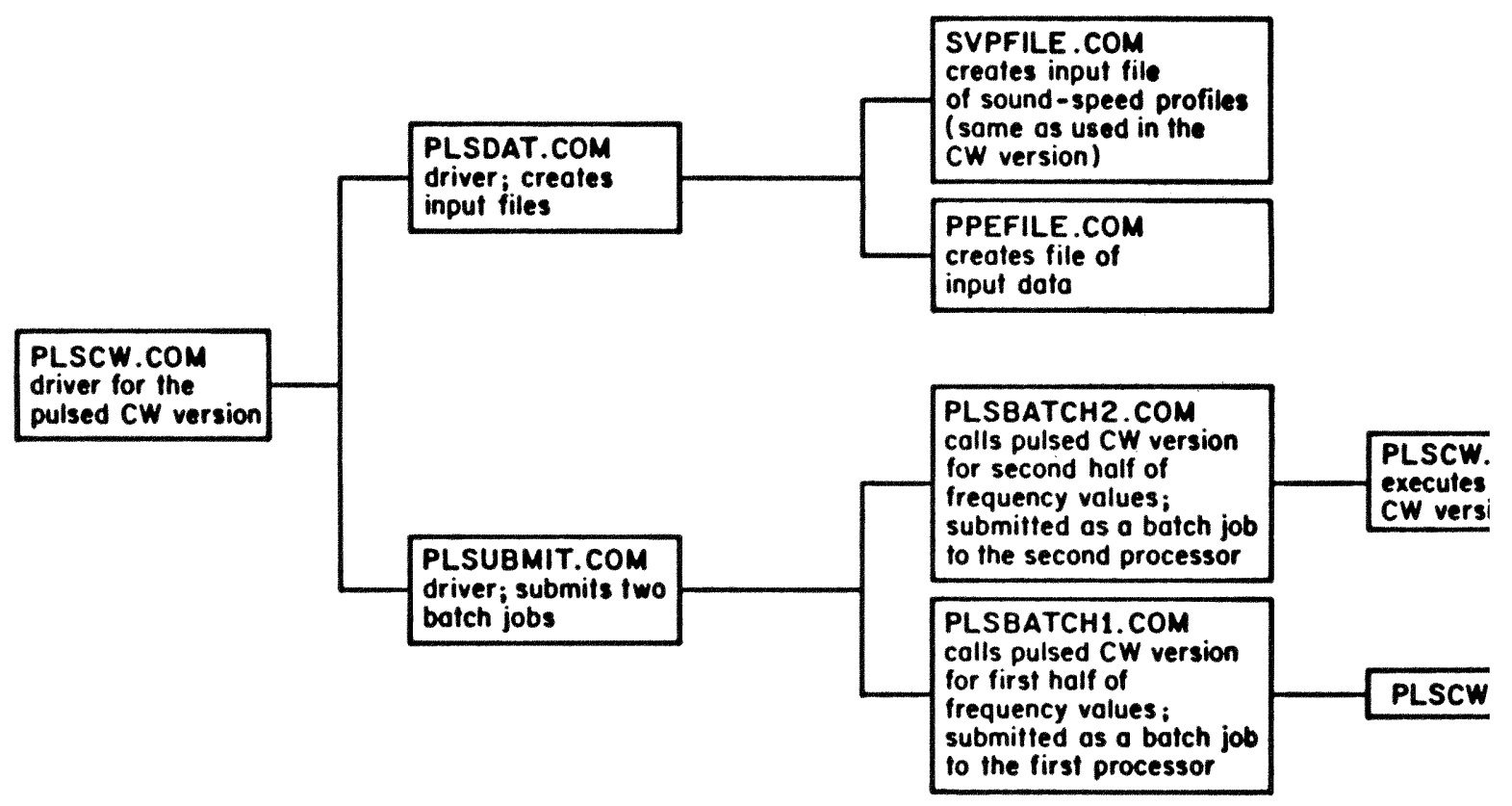

Figure 2. Out1ine of Model Structure (PSP CW Module). 


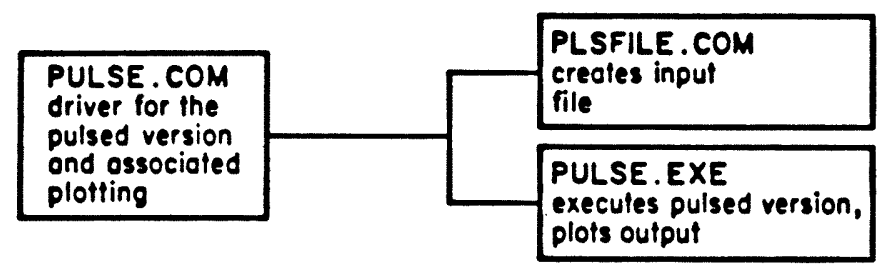

Figure 3. Outline of Model Structure (PSP Pulse Module).

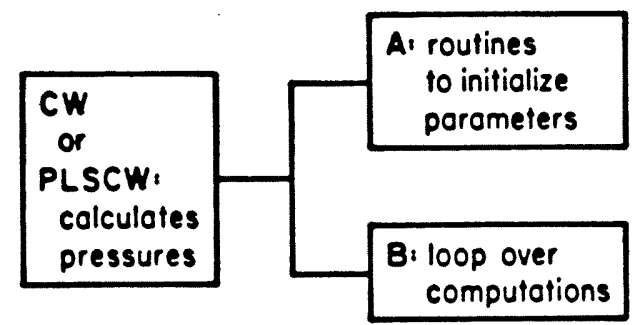

Figure 4. Source Code Structure for $\mathrm{CW}$ and PSP CW (PLSCW). 


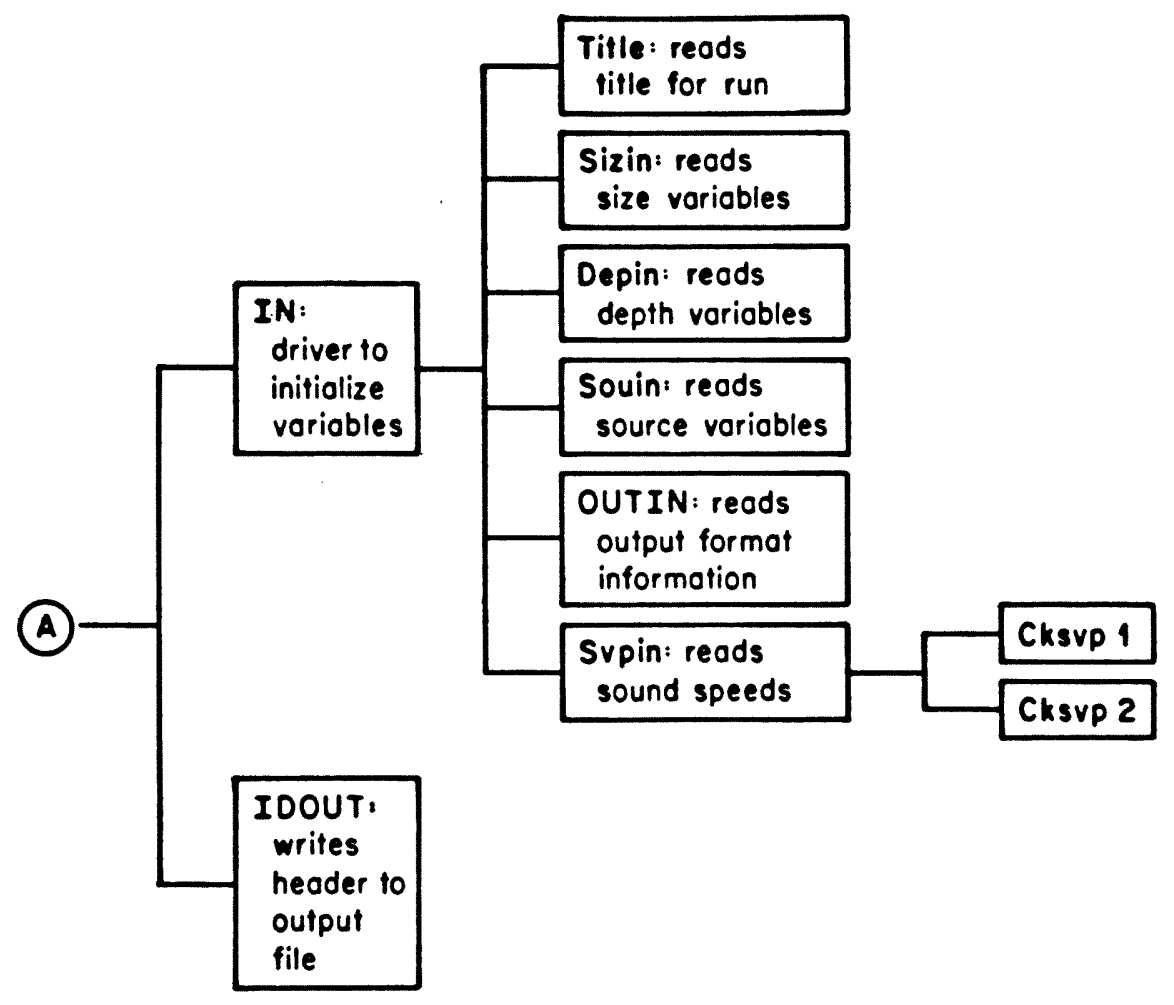

Figure 5. Inttializing Routines for CW Version. 


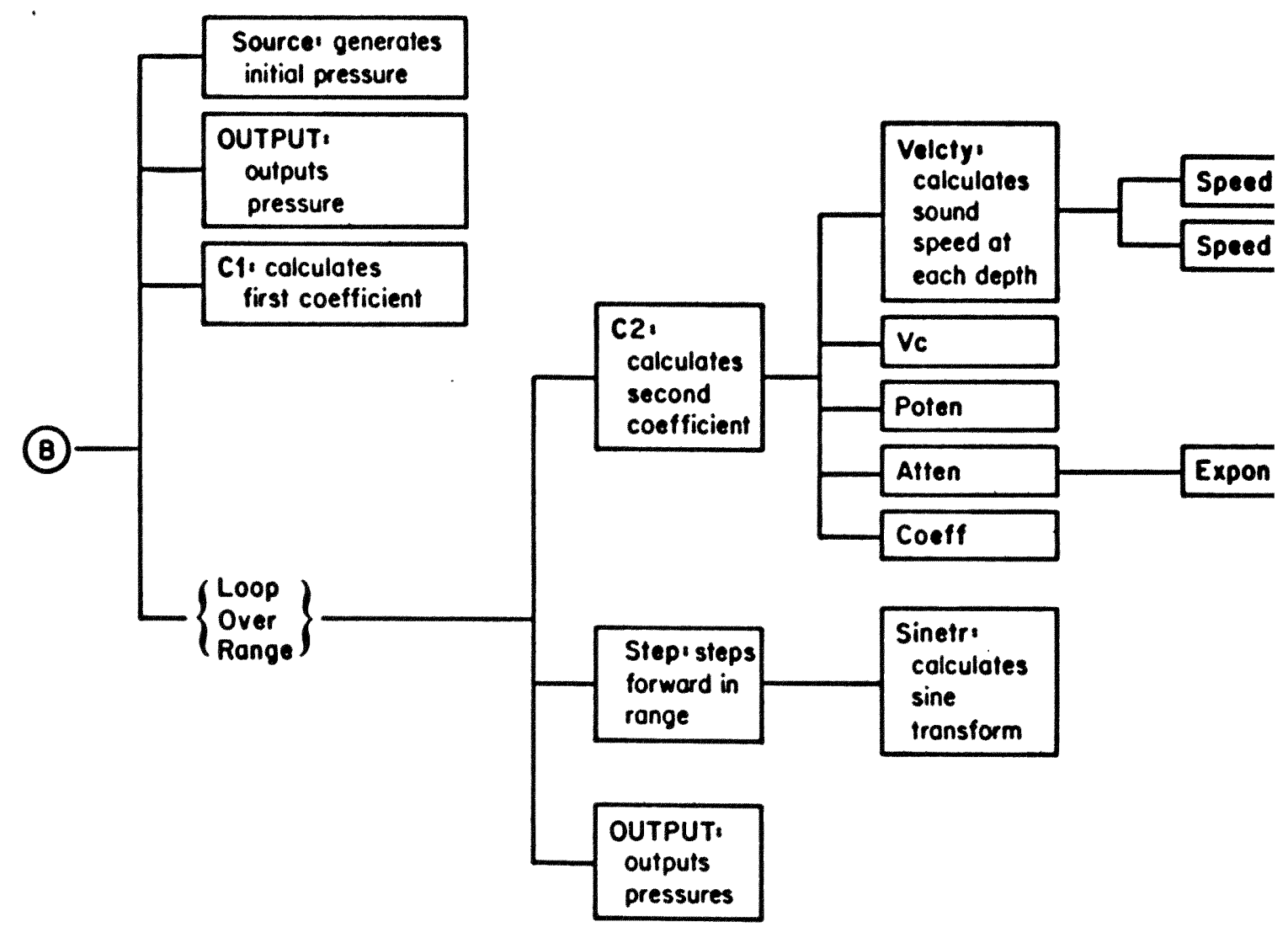

Figure 6. Calculation Routines for CW Version. 


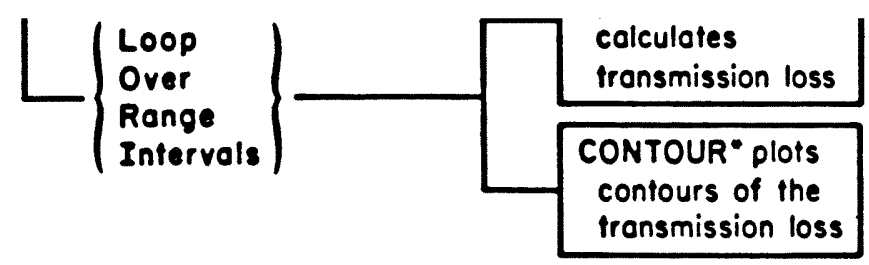

Figure 7. Schematic for Contour Plots for CW Version.

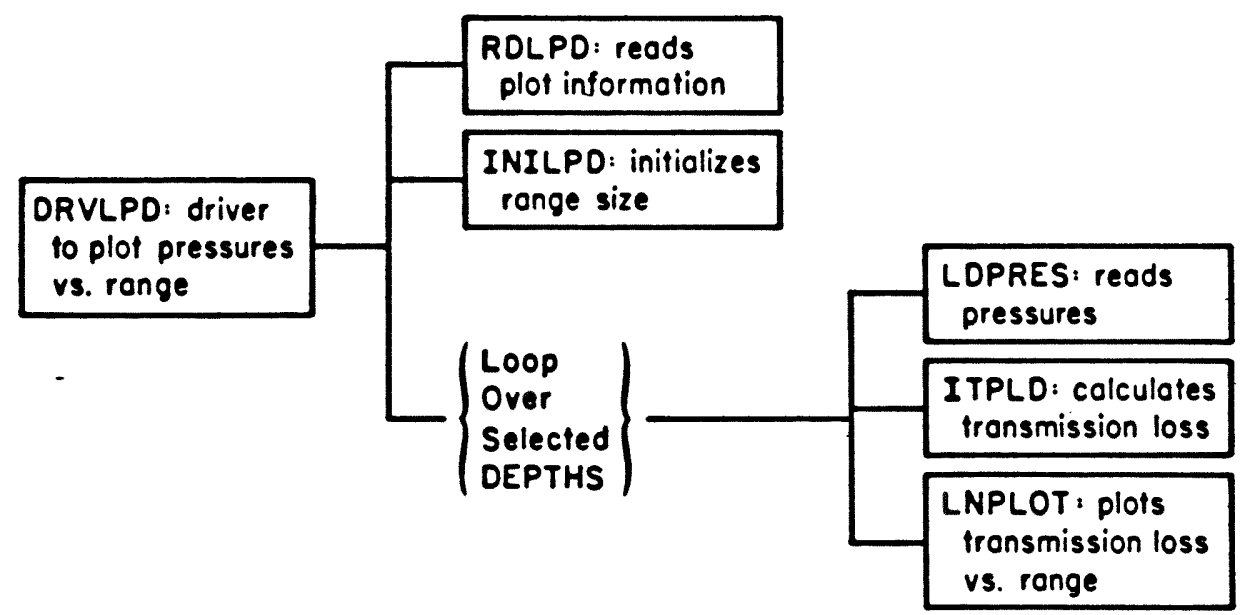

Figure 8. Schematic for Line Plots for CW Version. 


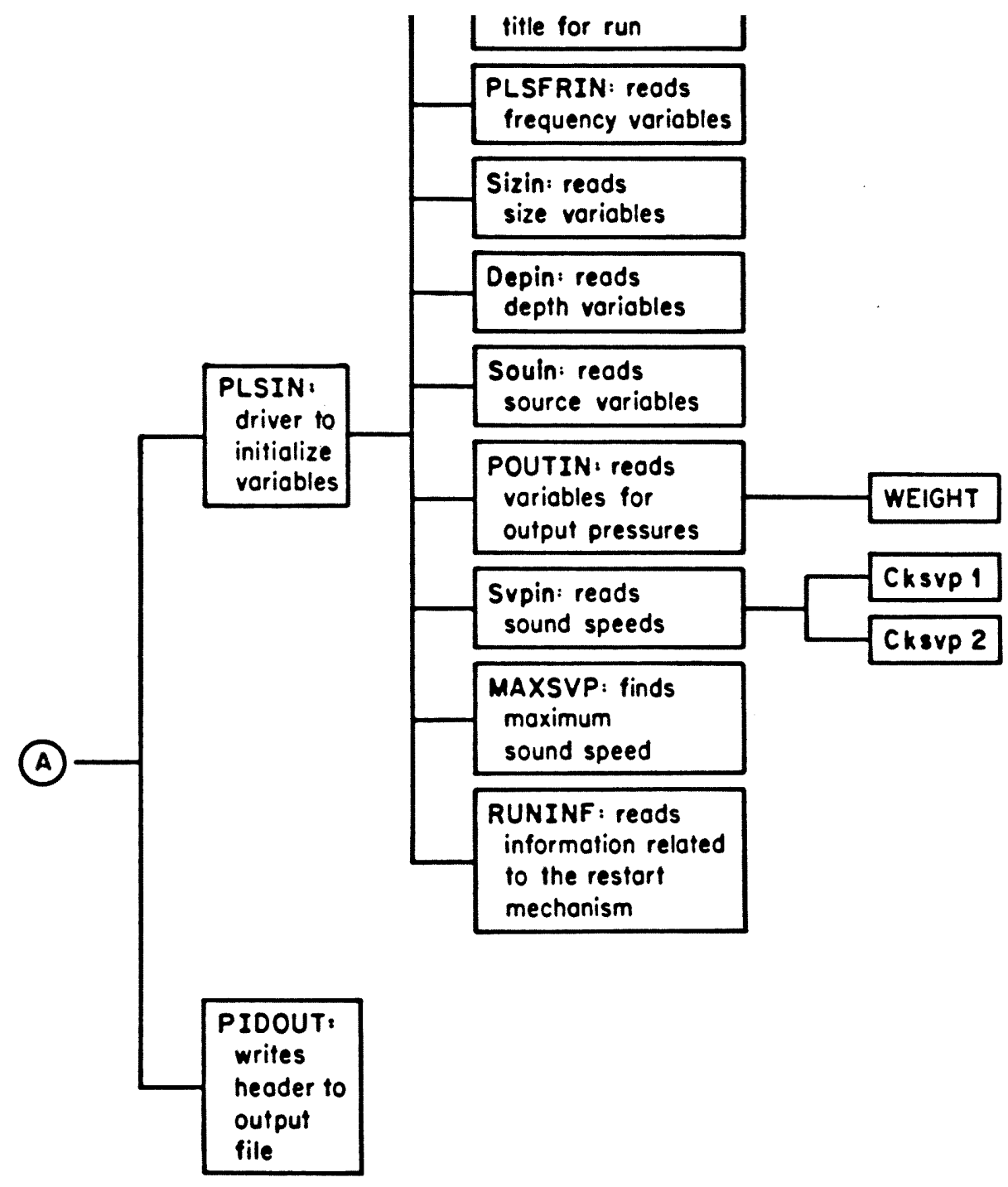

Figure 9. Initializing Routines for PSP CW Module. 


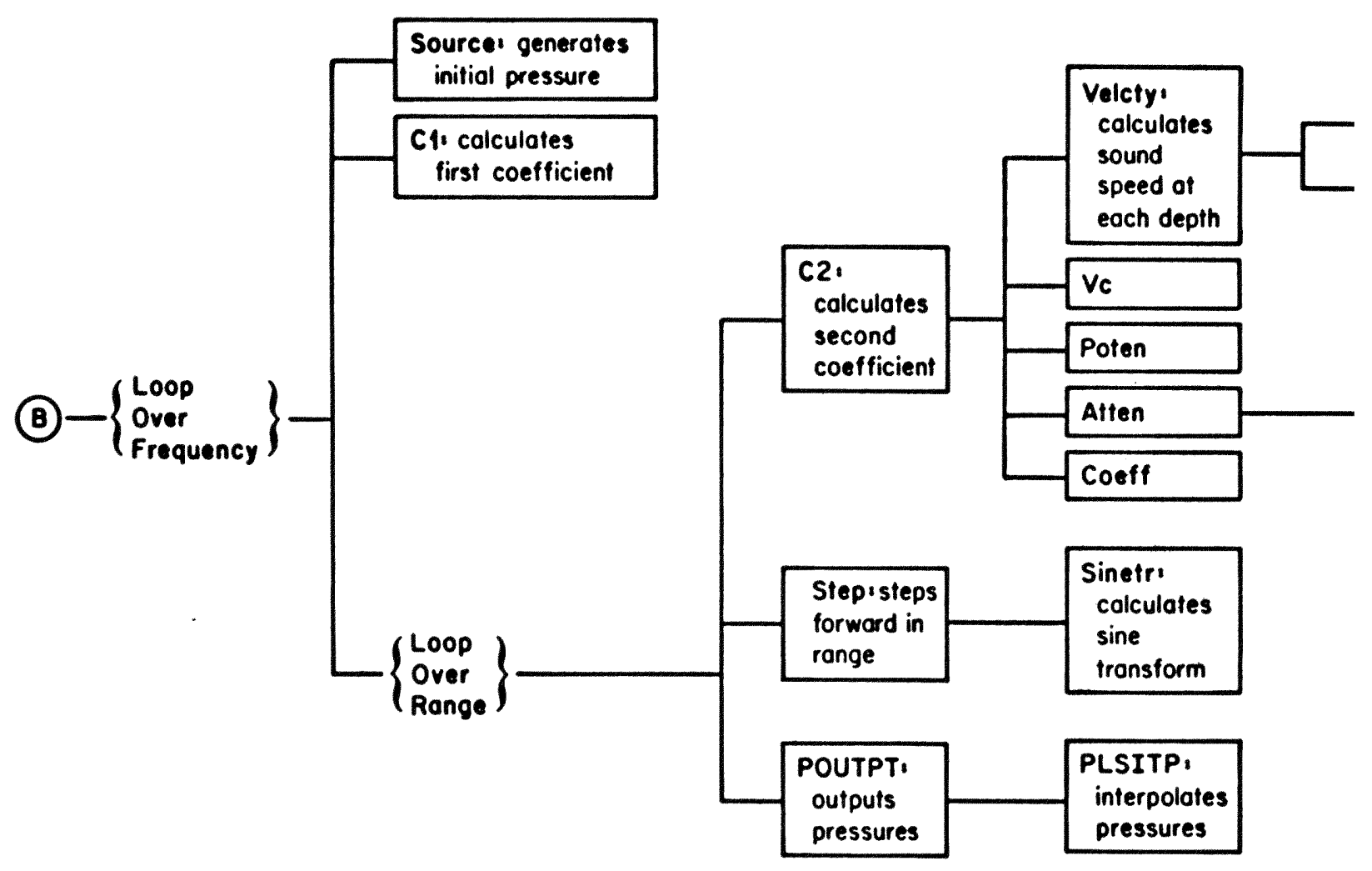

Figure 10. Calculation Routines for PSP CW Module. 


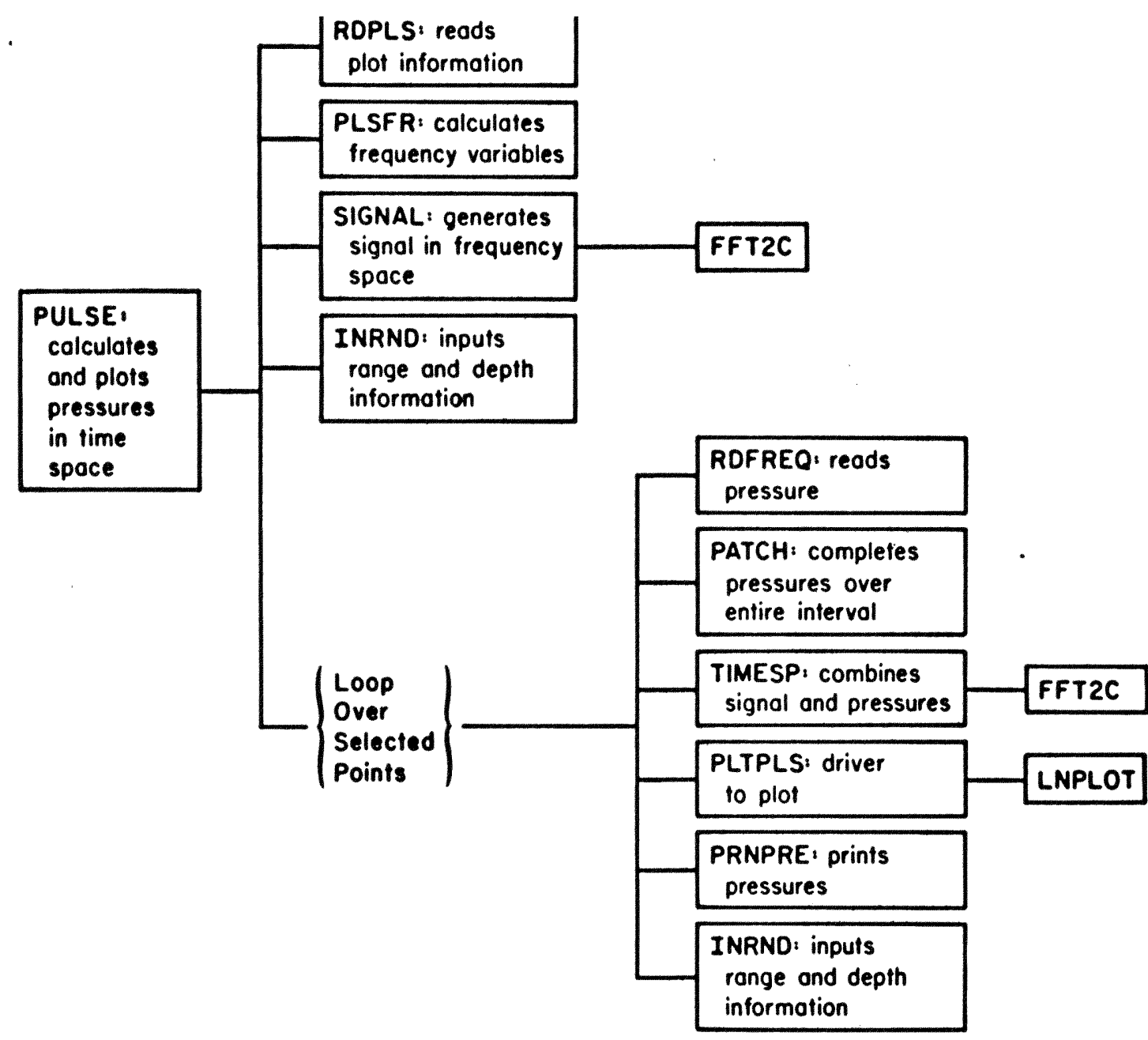


comparing the initializing routines (Figures 5 and 9). By comparing the computation routines in Figures 6 and 10, it is clear that the PSP CW expands the CW calculation one dimension further by the addition of the loop over frequencies. In all of these figures, the use of small letters means that the routine is exactly the same in both the PSP and CW versions. The asterisks in Figure 7 indicate that either IGL or UNI should be appended to the routine names, depending whether the IGL or Uniras plot routines are used. For example, if Uniras is used DRVCON*, LDARAY*, and CONTOUR* should be replaced by DRVCONUNI, LDARAYUNI, and CONTOURUNI.

The PSP Pulse module has one executable element, PULSE.EXE. The routines for this element are outlined in Figure 11.

B. Command Language Description

A command procedure is a file that contains a list of command language commands. Digital command language (DCL) is the system command language for the VAX/VMS computer system. This command language resembles a high level language in its ability to obtain input, write output, establish loops and conditional coding. In addition, DCL can perform system-related tasks like executing Fortran programs, copying files, appending one file to another, resetting system input or output devices, setting restarting location in case of system failure, and synchronizing two processes running in different processors. A11 of these available tools where used in the development of this model to offer an efficient, easy-to-use package for the non-technical user.

A primary feature of the model is that the CW version and both of the two modules for the PSP version are self-directed programs that 
allow the user to run them with a single command. The major DCL programs are drivers to routines that create files, execute the model and/or plot the results. The user is given different options that only require a $\mathrm{Y}$ (yes) or $\mathrm{N}$ (no) be entered.

User-friendly DCL programs that run in an interactive mode were developed to create each of the input files. The user does not have to get involved in the cumbersome and sometimes difficult task of creating complex Input files correctly. The programs prompt the user for all the pertinent information, including file name specification, and writes this information to a file in the appropiate format. In the prompts the units for input data are always indicated and maximum and/or minimum sizes whenever pertinent. The Fortran namelist facility was used to make files more readable in case the user needs to look at them.

DCL has different command levels and procedures can be nested by executing a command procedure from another command procedure. Every time this happens system input and output devices default to different values depending on the DCL level. Since this is an interactive model care had to be taken to reset system input and output to be the terminal.

A11 the information in the VAX-11 system is defined in terms of files. Files are organized in a tree structure where any node may contain files that are programs, data, text or directories. A file is named by giving it a file specification with the following format:

node: : device: [directory]filename.type; version

The network node, device, and directory names if not specified default to your current network, default disk and directory name. The 
directory name is a sequence of directory names where each represent a directory level (node in tree). A file type is one to three characters that usually identify the file in terms of its contents. The version is an integer assigned by the machine and incremented sequentially whenever a new version of a file is created. When describing files, version numbers are typically ignored. This organization was used to help the user and the maintenance programmer keep track of the source code program, object modules, executable modules, command procedures and data files. For the model the following default types where used:

. COM - designates a command procedure file to be executed with the a (execute procedure) command, or to be submitted for batch execution with the submit command.

.FOR - designates an input source file for the VAX-11 Fortran compiler. .OBJ - designates an object file created by the compiler.

.EXE - designates an executable file created by the linker.

For the data files, the user is prompted with suggested file types whenever a file name is entered. However, these are only recommendations and the user may choose any file type.

For example, the executable module for the $C W$ version fille name is:

$$
\text { AOML : :D5 : [OAD.GONZALEZ.PPEMAT]CW.EXE, }
$$

the source code for the main program of the PSP Pulse module file name is:

$$
\text { AOML : : :55:[OAD.GONZALEZ.PPEMAT] PULSE.FOR, }
$$

the command procedure that executes the PSP CW module file name is:

$$
\text { AOML : :D5 : [OAD.GONZALEZ.PPEMAT] PLSCW.COM • }
$$


(For more information on the file types of the data files see Section V: USER'S GUIDE - File Structures).

Programs in different nodes can have their protection set so that they can be executed by different users. Every time the user runs the model the programs stored in the program's directory node would be executed. In this particular case that node is AOML::D5:[OAD.GONZALEZ. PPEMAT]. This saves space since only one copy of each program must be stored. This copy can be accessed by many users at the same time. This feature also simplifies the maintenance since changes need to be done only at one place.

The model consists of two main parts: the $\mathrm{CW}$ version and the pulsed (PSP) version. The $C W$ version is a model for continuous wave sound propagation. It calculates the pressure field as a function of range and depth for a single frequency. Contour plots of transmission loss and line plots of transmission loss versus range can be obtalned. The program runs in an interactive mode in which input files are created, the model executed and results plotted. The user is also prompted to change the terminal mode to graphics and back to normal for the IGL plots. In the case of the UNIRAS contour plots, the progran sets the mode to graphics and allocates all the necessary files for the user.

The $C W$ version is run by executing the command language program CW.COM. Its structure is as follows.

- Give general directions to the user

- If user wants to run the CW model then

- QCWRUN.COM

Where: 
- The user is prompted to enter input information file name

- If the user needs to create the input information file then

- prompt the user to enter sound speed profiles (SVP) file name

- if the user needs to create the SVP file then

$$
\text { QSVPFILE.COM (creates SVP input file) }
$$

endif

- @PEFILE.COM (creates input information file)

Endif

- Go to a scratch node

- Create temporary DCL program to perform the model run using CW.EXE

- Ring be11 to indicate completion

-Return to user's node

- End

- If the user wants to plot transmission-loss contours then

- @CWCONTOUR.COM

Where:

- If the user needs to create the contour information file then

- QCONFILE.COM (creates the information file)

Endif

- If the user wants to plot UNIRAS contours then

- Run DRVCONUNI.EXE (creates contour plots)

Endif

- If the user wants to plot IGL color line contours then

- Run DRVCONIGL.EXE (creates contour plots)

Endif 
- Endif

- If the user wants line plots of transmission-loss then

- QCWDEPTH.COM

Where:

- If the user needs to create the information file then

- QLPDFILE.COM (creates the information file)

Endif

- Run DRVLPD.EXE (creates line plots)

- End

- Endif

- Exit

The PSP version is a model for pulsed sound propagation consisting of two modules, the PSP CW and PSP Pulse modules. The PSP CW module is similar to the $\mathrm{CW}$ version but performs the calculations for an interval of frequencies. At any given range and depth the PSP version calculates the pressure field as a function of time by transforming from frequency space. Plots can then be obtained. The PSP CW module runs in an interactive mode in which input files are created and batch jobs are submitted. (Running times are measured in hours.) Once the batch jobs are finished a second command language program (the PSP Pulsed module) can be executed which, interactively, creates needed specification files, calculates the pressure, and plots results.

The PSP version is run by executing two command language programs consecutively. Since the PSP CW module nust be run before PSP Pulsed, PLSCW.com is executed first. Its structure is as follows. 
- Go to a scratch node

- Give general directions to the user

- @PLSDAT.COM

Where:

- The user is prompted to enter input information file name

- If the user needs to create the input information file then

- prompt the user to enter SVP file name

- if the user need to create the SVP file then

QSVPFILE.COM (creates SVP input file)

endif

- QPPEFILE.COM (creates input information file)

Endif

- The user is prompted to enter output information file name

- Create temporary file with the names of the input and output files

- End

- @PLSUBMIT.COM

Where:

- Rename temporary file created by PLSDAT.COM with a unique name

- Initialize the number of frequencies for which pressures are calculated and stored, in each call to PLSCW.EXE

- Run LOOP.EXE (calculates the number of times each batch job should call PLSCW.EXE, and writes it out to a temporary file)

- Create temporary file for both batch jobs with frequency information 
- Submit PLSBATCH2, the batch job that calculates pressures for the second half of the frequencies, to be run by one processor

- Submit PLSBATCH1, the batch job that calculates pressures for the first half of the frequencles to be run by the other processor

- End

- Return to user's node

- Exit

PLSBATCH2.COM has the following structure:

- If Restart then go to appropriate restart location

- Set restart location

- Read input file name

- Read in initial loop value for the second half of the frequencies

- Read in the number of times PLSCW.EXE should be executed

- Do I = initial loop, total number of loops

- Create and execute DCL program to run PLSCW.EXE

- Overwrite value of initial loop in the temporary file to be $I+1$ (this is used for the restart mechanism)

- End Do

- Set restart location

- Delete temporary files

- End

PLSBATCH1.COM has the following structure:

- If Restart then go to appropriate restart location 
- Set restart location

- Read input file name

- Read in initial loop value for the first half of the frequencies

- Read in half the number of times PLSCW.EXE should be executed

- Do I = initial loop, half the number of loops

- Create and execute DCL program to run PLSCW.EXE

- Overwrite value of Initial loop in the temporary file to be $I+1$ (this is used for the restart mechanism)

- End Do

- Set restart location

- Delete temporary files

- Set restart location

- Synchronize with PLSBATCH2.COM (wait until it finishes)

- Set restart location

- Copy file with header and first frequencies to the PLSCW output file

- Do $I=1$, number of times PLSCW.COM was called by both batch jobs

- Append temporary PLSCW output file to the PLSCW output file

- End do

- Set restart location

- Delete all temporary files left

- End

When the batch jobs described above are completed, the PSP Pulsed module is run. PULSE.COM is executed to create the plot specification 
file, calculate the pressure as a function of time and plot the results. Its structure is as follows.

- Give general directions to the user

- User is prompted to enter Input information file name

- If the user needs to create the input information file then

- QPLSFILE.COM (creates input information file)

Endif

- Run PULSE.EXE (calculates the pulses and plots them)

- End

Sometimes the DCL programs had to create other temporary DCL programs or data files to run parts of the model. The creation of these temporary programs and files is transparent to the user. These temporary files are created in a scratch workspace in order to insure that the users' nodes are in no way affected when the model is run and that enough space is provided for these temporary files. The DCL command "set default" was used to change the user from one node to the other. The temporary files created are named using the process ID, the time (hour, minute, second) and a name. For example:

$$
\text { A204002A4205439BATCH1 .COM, }
$$

where A204002A4 is the process ID, 20 is the hour, 54 is the minutes, 39 is the seconds and BATCH1 is the name. In this scratch node, many users can run at the same time or the same user can submit several different batch jobs since these temporary files are given unique names. Once the program finishes using these files, they are deleted and execution is returned to the user's node. Even though this is all user-transparent, 
the user is advised that the node is being changed to insure that complete file name specifications are entered. This scheme for naming temporary files is used extensively in PLSUBMIT.COM, PLSBATCH1.COM and PLSBATCH2.COM, where it is primarily used to synchronize the two processors and to implement the restart mechanism.

The PSP version of the model runs in the range of 6 to 37 hours. There are many problems associated with running a program for such a long time. Most obvious ones are the waiting time and the fact that during such long periods the computer can fall and all the computation 1ost. To partially alleviate these problems a scheme was developed to submit two batch jobs to the two processors avallable, each to compute the pressures for half of the frequencies, with the ability to restart in case of system failure.

The two processors avallable in the VAX-11 are loosely coupled. This means they can be synchronized but each job is executed only by the processor to which it is submitted. The command procedure PLSUBMIT.COM creates temporary data files to indicate which frequencies are to be used in the computation by each batch job. The command procedure PLSBATCH1.COM computes the pressures for the first half of the frequencies and the command procedure PLSBATCH2.COM computes the pressures for the second half of the frequencies. Once PLSBATCH1.COM is finished with the computation it checks to see if PLSBATCH2.COM is also finished. If not, it will walt (synchronized) for it to finish. In case PLSBATCH2.COM is already finished, when PLSBATCH1 checks the queue an error condition is returned. This error is ignored, and the PLSBATCH1.COM continues in its execution. PLSBATCHl then combines the output files into only one file, deletes the temporary files, and 
notifies the user of its completion. The waiting time is cut in half although the execution (CPU) time stays the same.

In addition to this, a restart mechanism was embedded into the command language code. In case of a system failure the two batch jobs will recover with a minimal loss of computation. The restart option works as follows. The restart location is set at different places in the program. In case of system fallure, when the program recovers it goes to the last place where the restart location was set. The approach is to divide the program Into independent, sequential tasks. Once a task is completed the restart location is set to the next task.

The tasks into which the batch programs are divided are the following: (PLSBATCH1.COM performs these five tasks. PLSBATCH2.COM only performs tasks one and two).

(1) Running the model.

(2) Deleting temporary files.

(3) Synchronizing.

(4) Copying all the temporary output files to one output file.

(5) Deleting temporary output files.

When the batch jobs are submitted it is done with the restart option. A unique name prefix (ID_1) is send to the batch jobs as a parameter. This unique name prefix will differentiate the temporary files created for/by this job from the files of other similar jobs that could be submitted simultaneously.

First Task:

To run the model the batch job executes the following sub-tasks: 
(A) Reads the input information file name from a temporary file called 'ID_ 1 'NAME.TXT.

(B) Reads the starting loop number and the number of frequencles for which pressures would be computed in each call to PLSCW.EXE. These values are read from a temporary file created by PLSUBMIT.COM called 'ID_1'BEGFR1.VAR ('ID_1'BEGFR2.VAR for PLSBATCH2.COM).

(C) Read the total number of loops (calls to PLSCW.EXE) to be performed by this batch job. This value is read from temporary file created by LOOP.EXE called 'ID_1'LOOP.VAR.

(D) While (loop number is less than total number of loops) do

a) Create command procedure to run the model and write out the pressures to a temporary output file named with the unique name prefix and the loop number.

b) Change the starting loop number in 'ID_l'BEGFRl.VAR

('ID_1'BEGFR2.VAR) to be loop number +1 .

In case of system failure, when the program recovers it starts the computation at the loop number after the last loop number that was completed. This starting loop number has been updated in the 'ID_1'BEGFR1.VAR ('ID_1'BEGFR2.VAR) file each time the program completed a loop. If the system fallure occurred half way through the execution of the PLSCW.EXE, the temporary file that was half-way written is closed. When the program recovers, the computation restarts with the same starting loop number and the results are written out to a higher version of the same temporary output file.

The number of frequencies for which pressures are calculated in each execution of the model was set to 8 in PLSUBMIT.COM. Each 
execution of the PLSCW.EXE was run for 15 to 25 minutes for the frequency values and the number of points used in the sample computations. In the worst case, 25 minutes of computation could be 1ost. The number of frequencies for which values are computed in each call can be changed in PLSUBMIT.COM to the user's convenience and/or needs.

Second Task:

When deleting temporary files an "on error continue" is set indicating that if there is an error the program should continue. If there is a system fallure when the program is half-way through deleting the files, when it recovers and tries to delete a file that has already been deleted it stops on an error condition. Setting the "on error continue" avoids having the program stop under this circumstance. After the files are deleted, this condition is re-set to stop on an error condition.

Third Task:

The PLSBATCH1.COM batch job synchronizes with PLSBATCH2.COM batch job by waiting for it to finish. In case of a system failure and restart, the program re-checks to see if the other batch job is still in the queue. If PLSBATCH2.COM is still executing, PLSBATCH1.COM waits for it to finish. If PLSBATCH2.COM is already finished PLSBATCH1.COM continues with the execution. In case a system failure occurred after the signal was sent and before it was received, when the system recovers PLSBATCH1.COM it checks and finds that the other batch job is no longer in the queue and continues with the execution. 


\section{Fourth Task:}

PLSBATCH1 reads the main output file name from a temporary file created by PLSUBMIT.COM called 'ID_l'NAME.TXT. It then copies the first temporary output file (that has the disk header) to the main output file. In a loop structure it appends all of the other temporary output files in the correct order to the main output file. Because of the way that the VAX-11 file structure works it always selects the highest version of the files being copied or appended.

In case of system failure, when the program recovers it re-creates the main output file with the same name and a higher version.

\section{Fifth Task:}

This task is performed in the same manner as the second task.

The restart mechanism is all user-transparent. It was thoroughly tested using both artificial means and actual system fallures.

\section{Source Language Description}

The programming language used for the source code was VAX-11 Fortran 77. Fortran was chosen since it is the most common language used by the scientific community. Fortran 77 offers some of the capabilities needed for structured programuing, such as the while and the case statement. The most noticeable drawback is not having a strong variable definition. To partially overcome this, all variables used in the programs are explicitly declared. The namelist facility of Fortran was used for the input files making them more readable.

To design the model a combination of a bottom-up and top-down approach was used. As discussed earlier, the top-down design is widely 
recognized as the best approach. However, the complexity of this problem made a strictly top-down approach very difficult and thus a bottom-up approach was substituted at the beginning.

The model consists of 2 parts: (1) the computation for the continuous wave (CW) case; and (2) the pulse sound propagation (PSP) for an interval of frequency. Because of the difficulties involved in visualizing the model as a whole, the $\mathrm{CW}$ design was done first. Even visualizing the interaction of the various parts of the CW model was problematic, since there were at least three factors at work: range, depth and frequency. Therefore, small structured subroutines were designed to do the calculations which were later fit together. Each of these small subroutines performed only one task using a set of input variables to produce a set of output variables. The equations were divided into factors and a routine to calculate each factor was designed and developed.

A main input subroutine was designed to call a number of subroutines where each of these read the variables that are associated with a particular parameter. For example, variables associated with range are input in one routine, those associated with depth are read in another, etc. A routine was developed to generate the initial field of pressures for the simulation. These initial values are called source.

The bottom-up approach clarified dependency relations among such factors as depth, range, and frequency thus revealing how these factors interact and indicating the order in which the subroutines were to be called.

The main program was designed by considering the parts of the equation that were dependent on range. All the routines that were 
related to the initialization process, such as reading input information and generating the source, were placed outside of the range loop. Computations that were not dependent on range were also placed outside the loop while only those range dependent calculations were placed inside the 1oop.

Programs were designed to write the calculated values out to disk. Output of input parameters and the run identification was inserted outslde the computation loop. The programs to output the pressure values calculated at a particular range were added inside the 1oop.

Because of the large quantities of data it was impossible to write everything out to disk. The output program was changed to be selective on which values would be output to disk and a new Input routine was designed and developed. This routine is called from the main input subroutine and allows the user to select which values are to be written out to disk.

This smal1-routine approach not only clarified the interaction of the model but also allowed the scientists to assign specific values to different parts of the equations for testing. It made it very easy to generate the source values in different ways or change the bottom boundary conditions for different simulations. The only thing required was to write a subroutine to generate new source values or a different description for the absorbing layer (the attenuation coefficient) and link these new routines in the executable module instead of the old ones. This made the model flexible, expandable and very easy to change. Structure of Calculation Routines for CW Module

The CW executable module's main program is CW.FOR and its structure is as follows. 
- CALL IN

Where:

- The input information file name is read and the file opened

- CALL TITLE (reads descriptive identification for run)

- CALL SIZIN (reads variables related to sizes)

- CALL DEPIN (reads variables related to depth)

- CALL SOUIN (reads variables related to the source)

- CALL OUTIN (reads variables specifying the output format)

- Read sound speed profiles file name and open file

- CALL SVPIN (reads sound speed profiles)

Where:

- CALL CKSVPI (checks array dimensions)

- CALL CKSVP2 (checks the sound speed profiles to be in ascending order in range)

- Read contours output file name and open file

- If the number of depths to output is not zero then

- Read depth line-plots output file name and open file

Endif

- All this information is written out to the terminal

- CALL IDOUT (writes header of output disks files)

- CALL SOURCE (generates initial pressure field)

- GAUSS (Gaussian distribution function used to generate the source)

- CALL OUTPUT (writes pressure values to both output disk files)

- CALL $C_{1}$ (calculates first coefficient $c_{1}$ ):

Where:

$$
c_{1}(1)=\exp \left(\left((-1 * d r) /\left(2.0 * k_{0}\right)\right) *\left(\left((1 * P I) / z_{\max }\right) * * 2\right)\right)
$$


Where:

$$
\begin{aligned}
i \quad= & \operatorname{SQRT}(-1) \\
\mathrm{PI}= & \pi \\
\mathrm{dr}= & \text { range step } \\
\mathrm{k}_{0}= & (2.0 * \mathrm{PI} * \mathrm{f}) / \mathrm{c}_{0} \text { (reference wave number) } \\
& \text { Where: } \quad \mathrm{c}_{0}=\text { reference sound speed } \\
1 \quad= & \text { point index (1 } \leq 1 \leq \text { number of points in transform) } \\
z_{\text {max }}= & \text { total depth including artificial layer }
\end{aligned}
$$

- Do $r=1$, number of ranges

- Call $c_{2}$ (driver to calculate the second coefficient $c_{2}$ )

Where:

$$
\begin{aligned}
& c_{2}(1)=a(1) * \operatorname{EXP}\left(-1 * d r * k_{0} *(-(n * * 2-1) / 2)\right) \\
& \text { Where: } \\
& a(1)=\text { attenuation coefficient } \\
& \mathrm{n} \quad=\mathrm{c}_{0} / \mathrm{c}(\mathrm{z}, \mathrm{r}) \\
& \text { Where: } \\
& c(z, r)=\text { sound speed at the given depth, } z \text {, range, } r \\
& c_{0} \quad=\text { reference sound speed. }
\end{aligned}
$$


- CALL ATTEN (calculates the attenuation coefficient a(1))

Where:

$$
a(1)=\left\{\begin{array}{l}
1, \text { for points in water } \\
\exp \left(\operatorname{amp} * \mathrm{dr} r^{*} \exp (-((((\operatorname{ppts}(1)-\text { depth })-\right. \\
\mathrm{dif}) / \operatorname{dif} 3) * * 2))), \\
\text { for points in artificial layer }
\end{array}\right.
$$

Where:

$$
\begin{aligned}
\text { amp }= & -.15 * 3.28 \\
\text { zpts }= & \text { depth at each point } \\
\text { depth }= & \text { depth of basin } \\
\text { dif }= & z_{\text {max }}-\text { depth } \\
& z_{\text {max }}=\text { total depth with artificial layer } \\
\text { dif3 }= & 1 / 3 \text { dif }
\end{aligned}
$$

- EXPON (user defined exponential function used to calculate the attenuation coefficient)

- CALL COEFF (combines the results into the second coefficient)

- Ca11 Step (steps the solution forward one range)

Where:

$$
\operatorname{psi}(\mathrm{r}+\mathrm{dr}, \mathrm{z})=\mathrm{c}_{2} * \mathrm{FFT}^{-1} \quad\left(c_{1} * \operatorname{FFT}(\operatorname{psi}(\mathrm{r}, \mathrm{z}))\right)
$$

where:

$$
\begin{aligned}
& c_{1}=\text { first coefficient calculated in } c_{1} \\
& c_{2}=\text { second coefficient calculated in } c_{2} \\
& \text { FFT }=\text { fast Fourier transform (we use a sine } \\
& \quad \text { transform) } \\
& \text { psi }(r, z)=\text { pressure field at a given range, } r, \\
& \quad \text { depth, } z \\
& d r=\text { range step }
\end{aligned}
$$


- If range $r$ is one of the selected ones for output - CALL OUTPUT

\section{- Continue}

- Stop

- End

Structure of Plot Routines

Since large data sets are generated by the calculations, it became apparent that it would be necessary to develop graphics routines to not only display the final model results, but also to use in the debugging procedure. Two types of plots were required, contours and line plots. In order to insure flexibility, sophisticated drivers to the contour package and line-plot package routines were designed and developed. These options include aspects such as the length of the interval to be plotted (usually range), several options to label (or not label) each axis, selection of maximum and minimum values for line-plots, and the specification of contour levels. Whenever possible, natural associations were considered while developing the plot routines. For example, since the ocean surface is normally considered to be at the top of a plot, with the depth increasing downward, it was important to have an option of inverting the axis. This required that the user be able to flip the axes and values.

IGL library routines were incorporated in the line-plot graphics package (LNPLOT). Various options such as those allowing axis inversion were included, as well as an ability to normalize by a chosen value. This allows the user to compare a sequence of plots.

For the contour package, two different contour techniques, and thereby two different library packages, were used. The IGL contour 
routine was used to produce color-line contour displays, while UNIRAS was used to obtain raster-type color-filled contours.

These plot routines are run in an interactive mode and the user can select different options at the time the plots are run. Default values are provided for these routines. The selected plots are stored in segments and after the run the user can re-plot them, more than one at a time, to make comparisons or to get hard coples.

The driver programs for making transmission loss contours of depth versus range are: DRVCONUNI, the driver that uses the UNIRAS plot package to produce raster type color contours, and DRVCONIGL, the driver that uses the IGL plot package to produce color line contours. The DRVCONUNI and DRVCONIGL program modules have the same structure. Their structure is as follows.

- Ca11 RDCON (reads input information)

- Call INICON (calculates number of pressures to be read in per plot)

- Do for number of loops needed to plot all ranges

- Call LDARAYUNI (LDARAYIGL) (reads calculated pressures from disk and calculates transmission loss, loads matrix to be contoured)

- Call CONTOURUNI (CONTOURIGL) (package to plot contours)

- Print message and prompt user to hit "enter" for next plot

- End Do

- Stop

- End

The driver program for making line plots of the transmission loss is a function of range for specified depths is called DRVLPD. The DRVLPD executable module's main progran structure is as follows. 
- CALL RDLPD (reads input information)

- CALl INILPD (calculates number of pressure values to be read in per plot)

- Initialize plot routines

- Do for number of loops needed to plot all ranges

- CALL LDPRES (reads calculated pressures from disk)

- CALL ENCOD1 (encodes axis labels and prompt)

- Do While (selected plot depth is in the plot interval)

- CALL CHKDEP (check depth is in plot interval)

Where:

- Valid depth $=$ true

- If user wants to continue to next depth interval or it is first time through this loop then

- Re-sets index and depth values

- CALL ENCODE2 (encodes prompt with depth values for interactive plots)

- Valid depth = false

E1se

- If selected depth is not in range print message valid depth $=$ false

Endif

Endif

- If (valid depth) then

- CALL ITPLD (interpolates depth value and calculates transmission loss values to be plotted) 
- CALL ENCOD3 (encodes main label and message with number where plot would be store in the terminal)

- CALL LNPLOP (plot package to plot a iine). (See description for LNPLOT in this section.)

Endif

- Prompt user and read next depth for which values should be plotted

- End Do

- End Do

- End plot routines

- Stop

- End

The LNPLOT is the line-plot package that was designed and developed. It has the following structure:

- Call GSTART (routine that initializes graphics)

- Call LNPLOT (driver to line plot package)

Where:

- IF (flag for max, min value) CALL MAXMIN (determines maximum and minimum values in arrays to be plotted. Depends on flag if it would determine max and min for $X$ axis, for $Y$ axis or for both)

- Clear screen

- CALL WINORM (initializes window size for plot)

- CALL OPNSEG (IGL routine to open plot segment) 
- CALL LINCLR (IGL routine to set line color)

- CALL PLTLIN (plots the 1ine)

- CALL LINCLR (IGL routine to set axis color)

- Cal1 DRAXIs (draws the axis)

- CALL AXPOS (to determine X,Y of axis corner)

- If (flag for labels) then

- Ca11 TXTCLR (IGL routine to determine text color)

- Ca11 LABELS (writes out labels on plots)

- Call LABPOS (to determine labels positions)

Endif

- Return

- End

- Cal1 CLOSEg (IGL routine to close a segment)

- Cal1 GRSTOP (IGL routine to end the graphics IGL package)

Structure of Pulse Modules

Once the design for the $\mathrm{CW}$ was conceived and implemented, it was possible to expand the algorithms to a module that calculates the pressures for a band of frequency (PSP CW version). A large section of the PSP version has structure similar to that of the CW model and invokes the same subroutines for the executable calculations. Whereas the $\mathrm{CW}$ performs the calculations for only one frequency, the PSP uses a loop to calculate the values for several frequencies. 
A main input subroutine similar to the one used in the $C W$ version was designed and implemented with some variations to take into account the interval of frequencies. Computations were separated based on frequency dependence and a loop to perform the calculations for a band of frequency was added to the main program.

Due to two basic differences in the CW and PSP CW modules the PSP CW module was designed as an independent version, rather than using the $\mathrm{CW}$ version with an outer loop for frequencies. First, the running time for the CW version is between 15 and 20 minutes as opposed to 6 to 37 (or more) hours to run the PSP CW version. Because of these time constraints, the $C W$ version could be run in an interactive mode with a higher priority, whereas the PSP CW version would have to be submitted as a batch job. Second, and most important, is that the output of the programs are completely different. Since different quantities are calculated in the PSP CW version, the plots are different from the CW version, as also are the format of data output. In the CW version the main concern is the size of the output file, since scientists are usually interested in the transmission-loss throughout the entire range. Because the PSP CW version calculates pressures for an interval of frequencies (on the order of tens or hundreds), the number of values calculated is even larger. Being impractical to save all of the calculations, PSP $\mathrm{CW}$ was designed so that the information at all frequencies is saved at only selected points in range and depth. It is possible that the selected points do not correspond to grid points, introducing the necessity to interpolate the pressures using the surrounding grid points. 
Using the same "small routine" approach the new structure was designed and developed. The calls to the routines were then inserted at the appropiate places.

Due to the length of the PSP CW runs, a restart algorithm was developed to recover previous calculations in the event of a system fallure. When the restart mechanism was embedded in the DCL code the only modification required in the source code was to insert a call to another routine in the maln input subroutine. This added routine reads from a user-transparent file the frequencles for which the pressures are calculated in this run. This modification was this simple because the design of the model was flexible, expandable and well structured.

The PLSCW executable module's main program is PLSCW.FOR and its structure is as follows.

\section{- CALL PLSIN}

Where:

- It reads in input information file name and open file

- It reads in run information file name and open file

- It reads output file name and open file

- CALL TITLE (see: CW)

- CALL PLSFRIN (reads in variables related to frequency)

- CALL SIZIN (see: CW)

- CALL DEPIN (see: CW)

- CALL SOUIN (see: CW)

- CALL POUTIN (reads in variables that specify the output format)

- CALL WEIGHT (calculates relative weights rangewise and depth-wise for the selected ranges and depths. This is use to interpolate the pressure values) 
- Read sound speed profiles file name and open file

- CALL SVPIN (see: CW)

- CALL MAXSVP (finds maximun value in the sound speed profiles)

- CALL RUNINF (reads temporary file created by PLSUBMIT.COM that indicates frequencies for to be calculated by this execution of PLSCW)

- All this information is written out to the terminal

- CALL PIDOUT (writes header of output disk file)

- DO $\mathrm{f}=$ first frequency, last frequency

- CALL SOURCE (see: CW)

- CALL Cl (see: CW)

- DO $r=1$, number of ranges

- Ca11 $\mathrm{C}_{2}$ (see: CW)

- Ca11 Step (see: CW)

- If $r$ is a range value previous to a selected one for output

- Call STORE (stores pressures to be used in interpolation)

Endif

- If $r$ is a range value after a selected one for output then

- CALL POUTPT (writes pressures to output disk file)

- CALL PLSITP (interpolates pressure values for selected ranges, depths)

Endif

- Continue

- Continue

- Stop

- End 
Due to the clarity of the PULSE module of the pulsed version, a top-down design was used. First the input information is read in. The sinusoidal signal to be used for the source is generated. In a while loop the user enters the selected range and depth for which output is desired. The pulsed response is then generated and the real pressures versus time are plotted. The user is given the option to plot the phase and/or print the real pressure values as a function of time. The user is then prompted for another point or flag to end the program. The same line plot package develop for the $C W$ version is used for the plots in this module.

The PULSE executable module's main program is PULSE.FOR and its structure is as follows.

- Ca11 RDPLS (read input information)

- Call PLSFR (calculates values related to the frequency interval)

- Call SIGNAL (generates a sinusoidal signal for the first $n$ points as a function of tine over a given number of cycles and for a specified repetition period)

Where:

$$
\operatorname{sig}(1)=\operatorname{CMPLX}(a m p * \operatorname{SIN}(2 * \operatorname{PI}(i-1) * f 0 * d t), 0.0)
$$

Where:

$$
\begin{aligned}
& 1=\text { point index }(1,2, \ldots, n) \\
& \text { amp }=\text { amplitude of the sinusoidal signal } \\
& \text { SIN }=\text { sine function } \\
& \text { PI }=3.1416 \\
& \text { fO }=\text { carrier frequency } \\
& d t=\text { delta time for pulsed calculation }
\end{aligned}
$$

Patch with zeroes for $n+1$, to number of points in signal 
- CALL FFT2C (transform signal to frequency space - FFT2C is an IMSL routine to compute the FFT of a complex value)

- CALL INRND (prompt user for a choice of ranges and depths selected in the run of the pulsed CW version or set end flag)

- Do while (not end)

- CALL RDFREQ (reads pressure values for all the frequencles for the selected range, depth)

- CALL PATCH (it sets an array with the pressures as follows:)

$$
p(f)= \begin{cases}0, & \text { for } \mathrm{f}<\mathrm{f} 0-1 / \mathrm{tau} \\ 1 / \mathrm{SQRT}(\mathrm{r}) * \operatorname{EXP}\left(i * 2 * \mathrm{PI} \mathrm{f}^{*} \mathrm{r} *(1 / \mathrm{c0}-1 / \mathrm{cmax}) * \mathrm{pr}(\mathrm{f})\right), \\ \text { for } \mathrm{f} 0-1 / \mathrm{tau} \leq \mathrm{f} \leq \mathrm{f} 0+1 / \mathrm{tau} \\ 0, \quad \text { for } \mathrm{f}>\mathrm{f} 0+1 / \text { tau }\end{cases}
$$

Where:

$$
\begin{aligned}
& \mathrm{f} \quad \text { frequency value } \\
& \mathrm{f} 0 \quad=\text { carrier frequency } \\
& \mathrm{tau} \quad=\text { pulse length } \\
& \mathrm{r} \quad=\text { range } \\
& \mathrm{pr}(\mathrm{f})=\text { output from the pulsed CW model (PLSCW) } \\
& \mathrm{c}_{\text {max }} \quad=\text { maximum sound speed value } \\
& \mathrm{c}_{0} \quad=\text { reference sound speed }
\end{aligned}
$$

- CALL TIMESP (combines signal transform and pressure in frequency space and finds real pressure and phase as a function of time)

Where:

Do $i=1$, number of points

$$
p(i)=\operatorname{CoNJG}(p(i) * \operatorname{sig}(i))
$$




\section{Continue}

Cal1 FFT2C (to transform $p$ values to time space)

Do $1=1$, number of points

$$
\begin{aligned}
& \operatorname{pr}(1)=\text { Real part of }(\operatorname{CONJG}(p(1)) / \text { number of } \\
& \text { points })
\end{aligned}
$$

Continue

Where:

$$
\mathrm{pr}=\text { real pressures as a function of time }
$$

- CALL PLSPLT (plots the pulsed and the phase)

- Calculates start time

- Calculates times for the $X$ axis and normalizes real pressures to be plotted in the $\mathrm{Y}$ axis

- Encodes labels

- Initializes graphics

- CALL LNPLOT

- If (user wants to plot the phase) then

- Ca11 LNPLOT

Endif

- End plot routines

- If (user selected to be prompted for optional print out) then

- Prompt user (yes or no)

- If (user wants this pressures values to be printed out) then

- CALL PRNPRE (prints out to a file the real pressures as a function of time for a selected range, depth 
Endif

Endif

- CALL INRND

- If (not end) then

- Rewind file

- CALL SKIPHD (skips file header)

\section{Endif}

- End do

- Stop

- End 
A. How To Run The Model

There are two versions of the PPEMAT model, the continuous wave (CW) version for modeling pressure flelds for a single frequency, and the pulse (PSP) version for pressures generated by a band of frequencies. PPEMAT is self-directed and runs in an interactive mode. The user is prompted and given instructions for entering all required information. A file naming scheme is suggested throughout the model, although the user is not required to adher to these suggested names. Additional information about the naming of files is contained in Section B: File Structure. Both versions of the model require input Information regarding the physical environment to be modeled and both produce graphics output to display the results. The user is prompted for plotting parameter specifications in order to create input files for the graphics routines. Explicit instructions are given for the different choices of plotting formats. Default values are always available, and it is suggested that initially the user run the plot routines with the defaults values.

\section{CW VERSION:}

The PPEMAT CW version calculates the pressure field for all depthrange points for a single frequency. Since the number of grid points used for the calculations can be very large, the user has the option of outputing only a subset of the calculated values to a disk file. There are two types of plots used to display the results, contour plots in range and depth of the transmission-loss values obtained from the pressures, and line plots of the transmission-loss versus range for a 
selected depth. The executable part of the $\mathrm{CW}$ version runs in a command mode.

To run the $C W$ version the user enters

C[ directory $] \mathrm{CW}$

The first prompt requires the user to create a sound speed data file or provide the name of a previously created file. Upon choosing to create the file, the user is prompted for the necessary data. This file contains the sound speed values as a function of depth for one or more ranges. These data are described below.

Number of sound speed profiles (at least one).

Number of points in the sound speed profiles.

Depth in meters at each point in the sound speed profiles.

For each sound speed profile the user must provide the range in kilometers and the sound speed in meters/seconds for each depth point.

These sound speed profiles are written to a file that can be used again on additional runs, or in a run of the pulsed version which requires the same input information. In the event that a sound speed data file already exists the user only needs to provide the name of the file.

The $C W$ version then requires the user to create a run information file or provide the name of a previously created file. This file contains the following information.

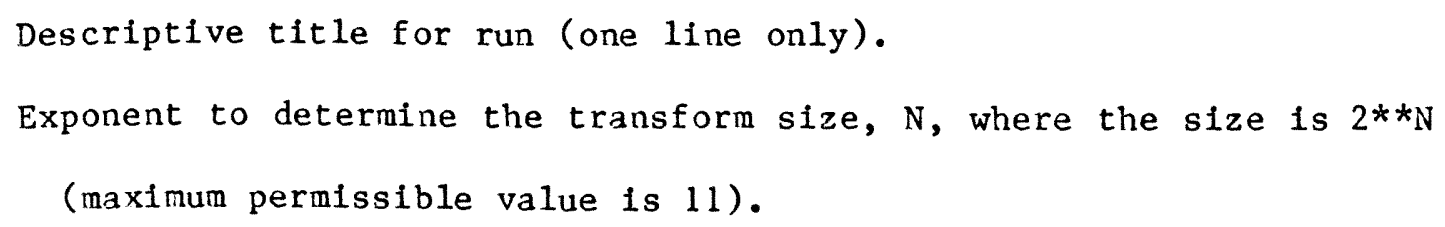


Maximum range of propagation in kilometers.

Range step (delta $r$ ) in meters. (The first range for calculation is always equal to the range step.)

Depth of basin in meters.

Bottom factor (default value of $4 / 3$ ) for the artificial layer.

Source depth in meters.

Acoustic wave frequency in hertz.

Reference sound speed in meters/second.

Depth index interva1, $k$, for output. This means that data is written to disk every $k$ th point starting with the first point. For example, if 8 is entered, the pressure value for depths with index $1,9,17, \ldots, 2^{* * \mathrm{~N}}-\mathrm{k}+1$ will be output to disk file.

Range index interval, $k$, for output. This means that data is written to disk every $k$ th range point starting with the first range. For example, if 3 is entered, the pressure values for ranges with Index $1,4,7, \ldots$, maximun range (in meters)) / range step $-k+1$ wil1 be output.

Number of selected depths values for which the transmission-1oss versus range will be plotted. (If 0 is entered, no output depth file is created.)

Selected depth values for the transmission-loss versus range plots. File name for the output file with the pressures to be contoured. File name for output file with the pressure at selected depths.

At this point the mode1 is actually executed. Upon completion the user is notified by a bell.

The user then has the option of plotting transmission loss contours either by using the raster type plots or the line contour routines. For 
each case a self-explanatory plot information file must be created, or the name of a previously created file given. When the fille is created the user must provide the following information.

Device number (this is the type of Tektronics terminal, e.g., 4113, 4010, or 4662).

Number of contour levels. If a negative number is entered the absolute value is used for the number of contours and the levels are determined by the program (evenly spaced between the maximum and minimum). If a positive number is entered the user must provide the beginning level and the increment between contours. Range to be plotted. If a positive number is entered each contour plot has that length in kilometers on the $x$ axis. For example, if the maximum range calculated is $500 \mathrm{~km}$ and the user enters $125 \mathrm{~km}$ for the range to be plotted then four segments, each $125 \mathrm{~km}$ in range, would be produced. If 0 is entered, the program calculates the range of the plots.

Plot format information including a choice of labeling the axes, plotting tick marks, drawing a box around the plot, plotting the grid lines.

The file name containing the pressures created above.

The user then has the option of making line plots of the transmission loss as a function of range for the previously specified depths. Upon choosing this option the user must provide the following information or provide a file name previously created.

File name for the plot information.

Device number. 
Plot format information including a choice of labeling the axes, selecting the maximum and minimum values for the axes, plotting tick marks.

The file name containing the pressures created above.

\section{PULSE VERSION:}

The PULSE version calculates the time history of the pressure received at any given point as the result of an input signal. Since the number of calculations is very large, this version of the model was divided into two command modules, the PSP CW module and the PSP Pulsed module. The PSP CW module calculates the pressures over band of frequencies while the PSP Pulse module continues these values with a description of the source signal and transforms from frequency to time space.

The PSP CW module essentially performs the calculations of the CW version over many frequencies. Since this module can require large CPU times, the executable part of the PSP CW module runs in a batch mode. The number of calculations resulted in a design to output to disk the presures only at selected points. The user must specify the range and depth values of interest. The only output from the PSP CW module are the pressures stored on a disk file.

The PSP Pulse module reads the calculated pressure, uses input information on the signal, and combines these through Fourier transforms to produce a time history of the real pressures and phases at the selected points. The output of this module consists of line plots of the pressure and phases as a function of time at each selected spatial point. 
To run the PSP CW module the user enters

$$
\text { [ [ directory] PLSCW }
$$

The user must create a sound speed data file or provide the name of a previously created file. A description of this file is provided above in the discussion of the $C W$ version. The PSP CW module prompts the user for the following information.

Descriptive title for run (one line only).

Repetition period in seconds.

Carrier frequency in hertz.

Number of cycles.

Number of samples per cycle.

Exponent to determine the transform size, $N$, where the size is $2 * *_{N}$ (maximuin permissible value is 11 ).

Maximum range of propagation in kilometers.

Range step $(\Delta \mathrm{r})$ in meters.

Depth of basin in meters.

Bottom factor (default value of $4 / 3$ ) for the artificial layer.

Source depth in meters.

Reference sound speed in meters/second.

Number of different ranges for recelvers (where the pressures are to be output).

Number of different depths for receivers (where the pressures are to be output).

Range in kilometers for each reciever.

Depth in meters for each receiver. 
At this point the PSP CW module submits the necessary batch jobs to both processors.

Upon completion of the calculations by the PSP CW module (run time depends on frequency interval and range), the user executes the PSP Pulse module of the PULSE version. This module calculates and plots the real pressures at the selected points.

To run the pulsed module the user enters

\section{Q [ di rectory] PULSE}

The user must create a plot information file or provide the name of a previously created file. To create the file the following information is required.

File name for the plot information.

Amplitude of the sinusoidal signal.

Device number.

Plot format information including a choice of labeling the axes, selecting the maximum and minimum values for the axes, plotting tick marks.

A cholce of plotting the phase at a given receiver.

A choice of printing the pressure values at a given receiver.

File name for disk file containing the calculated pressures.

\section{B. File Structure}

Al1 of the files in the PPEMAT model have the following file name specification:

[Directory]Filename. Filetype 
A file naming system was developed to differentiate among files and among runs. It is suggested that the user use the same file name for all of the files in a particular run and differentiate among them with the file type. Each time the user is prompted to enter a file name, the file type is suggested. For example:

"ENTER CONTOURS PLOTS' INFORMATION FILE NAME ( [DIRECTORY]FILENAME.CON)"

where:

CON is the suggested file type.

The file name specification should always include the user's directory since the model is run in a different node ([OARS.PPEMAT] in this case).

The three types of files used in the PPEMAT model can be classified as files that are created by DCL programs, files created by executable modules, and a print file. The various files are described below.

1) Files that are created by DCL command programs. These files are formatted files and are used to specify input information to the model. An example of an open statement in a module that reads a file of this type is

OPEN (UNIT $=$ 非,FILE $=$ f ilename, STATUS = ' OLD' , FORM= ' FORMATTED', READONLY $)$

Files types with this format are the following:

filename.SVP
filename.DAT
filename.CON
filename.LPD
filename.DAP 


\title{
filename.VAR
}

filename.PLS

2) Files that are created by the executable modules. These files are unformatted and contain the results of the calculations in the model. An example of an open statement in a module that reads a file of this type is

OPEN (UNIT $=\equiv \equiv$, FILE=f 1 l lename, STATUS= 'OLD' , FORM=' UNFORMATTED' , READONLY $)$

File type with this format are the following:

\author{
filename.PRE \\ filename.DEP \\ filename.RND
}

3) The file filename.PRN. This is a formatted output file of the real pressures as a function of time to be used to obtain a print of the values. To get a print out of this file the user enters

\section{PRINT filename.PRN}

The specific content of each of the files is described in the following tables. Each file is described by its name, the program which creates the file, the routine where it is opened, the sequence of read statements necessary to read the entire file, and a brief description of the contents. Following the tables, a file structure glossary is provided which contains an alphabetical listing of the variable names contained in the read statements and a brief description of their usage. 
Table 1: File Description, CW Model.

\begin{tabular}{|c|c|c|c|c|c|}
\hline Name of File & $\begin{array}{l}\text { Program Which } \\
\text { Creates File }\end{array}$ & $\begin{array}{l}\text { Opening } \\
\text { Routine }\end{array}$ & $\begin{array}{l}\text { Routines } \\
\text { Which } \\
\text { Read File }\end{array}$ & Read Statements & Contents \\
\hline filename.SVP & SUPFILE.COM & IN.FOR & SVPIN.FOR & $\begin{array}{l}\operatorname{READ}(\#, \operatorname{SVP}) \\
\operatorname{READ}(\#, *)(Z(I), I=1, N P T S V P) \\
\text { for } I=1, N S V P \\
\operatorname{READ}\left(\#,,^{*}\right) \operatorname{RNGSVP}(I) \\
\operatorname{READ}\left(\#,{ }^{*}\right)(\operatorname{VELSVP}(J, I), J=1, \operatorname{NPTSVP}\end{array}$ & $\begin{array}{l}\text { Values of the sound speed } \\
\text { as a function of depth and } \\
\text { range. }\end{array}$ \\
\hline \multirow[t]{5}{*}{ filename.DAT } & PEFILE.COM & IN. FOR & $\begin{array}{l}\text { TITLE.FOR } \\
\text { SIZIN.FOR }\end{array}$ & $\begin{array}{l}\operatorname{READ}\left(\#, ' A 80^{\prime}\right) \mathrm{ID} \\
\operatorname{READ}(\#, S I Z E)\end{array}$ & $\begin{array}{l}\text { User-provided specifica- } \\
\text { tion information for a } \\
\text { particular run. }\end{array}$ \\
\hline & & & DEPIN,FOR & READ( \#, BOTTOM) & \\
\hline & & & SOUIN.FOR & READ (\#, SOURCE) & \\
\hline & & & OUTIN.FOR & 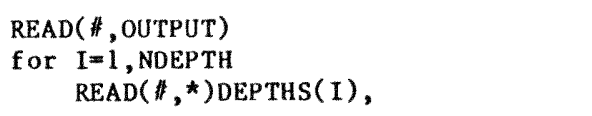 & \\
\hline & & & IN.FOR & 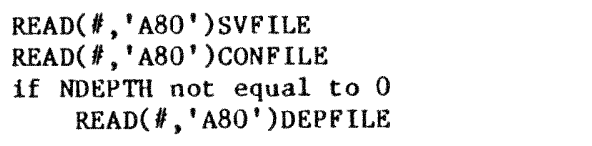 & \\
\hline \multirow[t]{2}{*}{ filename.PRE } & CW.EXE & RDCON.FOR & RDCON.FOR & $\begin{array}{l}\text { READ(\#) ID ,N, DR , NRANGE ,DEPTH, } \\
\text { ZMAX, ZS, CO, F ,PLZINV ,P LRINV }\end{array}$ & $\begin{array}{l}\text { Values of the pressure } \\
\text { needed for the contour } \\
\text { plots. }\end{array}$ \\
\hline & & & LDARAY . FOR & 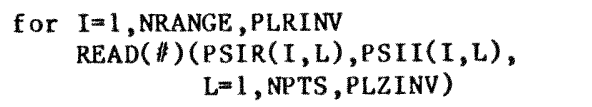 & \\
\hline
\end{tabular}


Table 2: File Description, CW Mode1 (continued)

\begin{tabular}{|c|c|c|c|c|c|}
\hline Name of File & $\begin{array}{l}\text { Program Which } \\
\text { Creates File }\end{array}$ & $\begin{array}{l}\text { Opening } \\
\text { Routine } \\
\end{array}$ & $\begin{array}{l}\text { Routines } \\
\text { Which } \\
\text { Read File }\end{array}$ & Read Statements & Contents \\
\hline \multirow[t]{2}{*}{ f 1 lename. DEP } & CW.EXE & RDLPD.FOR & RDLPD.FOR & $\begin{array}{l}\operatorname{READ}(\#) \text { ID }, \mathrm{N}, \mathrm{DR}, \mathrm{NRANGE}, \mathrm{DEPTH}, \\
\mathrm{ZMAX}, \mathrm{ZS}, \mathrm{CO}, \mathrm{F}, \mathrm{PLRINV}, \mathrm{NDEPTH} \\
\operatorname{READ}(\#)(\mathrm{DEPTHS}(\mathrm{J}), \mathrm{J}=1, \mathrm{NDEPTH}) \\
\operatorname{READ}(\#)\left(\mathrm{DINDEX}(\mathrm{J}), \mathrm{J}=1, \mathrm{NDEPTH}{ }^{*}\right)\end{array}$ & $\begin{array}{l}\text { Values of the pressure } \\
\text { needed for the line plots } \\
\text { of transmission loss. }\end{array}$ \\
\hline & & & LDPRES . FOR & 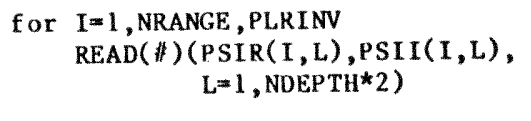 & \\
\hline fillename.CON & CONFILE.COM & RDCON . FOR & RDCON.FOR & $\begin{array}{l}\text { READ( \#, CONTUR) } \\
\operatorname{READ}\left(\#, ' A 80^{\prime}\right) \text { CONFILE }\end{array}$ & $\begin{array}{l}\text { Specification information } \\
\text { for the contour plots. }\end{array}$ \\
\hline f $₫$ lename.LPD & & & RDLPD.FOR & 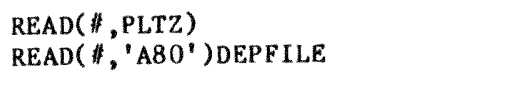 & $\begin{array}{l}\text { Specification information } \\
\text { for the line plots of } \\
\text { transmission loss. }\end{array}$ \\
\hline
\end{tabular}


Table 3: File Description, Pulsed Mode1.

\begin{tabular}{|c|c|c|c|c|c|}
\hline Name of File & $\begin{array}{l}\text { Program Which } \\
\text { Creates File }\end{array}$ & $\begin{array}{l}\text { Opening } \\
\text { Routine }\end{array}$ & $\begin{array}{l}\text { Routines } \\
\text { Wh1ch } \\
\text { Read F1le }\end{array}$ & Read Statements & Contents \\
\hline f1lename.SVP & SVPFILE.COM & PLSIN.FOR & SVPIN.FOR & $\begin{array}{l}\operatorname{READ}(\#, \operatorname{SVP}) \\
\operatorname{READ}(\|, *)(Z(I), I=1, \operatorname{NPTSVP}) \\
\text { for } I=1, \operatorname{NSVP} \\
\operatorname{READ}(\|, *) \operatorname{RNGSVP}(\mathrm{I}) \\
\operatorname{READ}(\|, *)(\operatorname{VELSVP}(\mathrm{J}, \mathrm{I}), \mathrm{J}=1, \operatorname{NPTSVP})\end{array}$ & $\begin{array}{l}\text { Sound-speed values; same } \\
\text { file as used in the CW } \\
\text { model. }\end{array}$ \\
\hline \multirow[t]{6}{*}{ filename.DAP } & PPEFILE.COM & PLSIN.FOR & $\begin{array}{l}\text { TITLE.FOR } \\
\text { PLSFRIN.FOR }\end{array}$ & $\begin{array}{l}\operatorname{READ}\left(\#, \text { ' }^{\prime} 80^{\prime} \text { ') ID }\right. \\
\operatorname{READ}(\#, \text { PLSFRE) }\end{array}$ & $\begin{array}{l}\text { User-provided specifica- } \\
\text { tion Information for a } \\
\text { particular run. }\end{array}$ \\
\hline & & & SIZIN.FOR & $\operatorname{READ}(\#, S I Z E)$ & \\
\hline & & & DEPIN.FOR & READ(\#,BOTTOM) & \\
\hline & & & SOUIN.FOR & READ (", SOURCE) & \\
\hline & & & POUTIN.FOR & 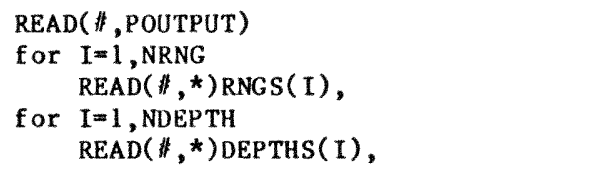 & \\
\hline & & & PLSIN.FOR & READ(\#, ' $\left.\mathrm{A} 80^{\prime}\right)$ SVF ILE & \\
\hline fllename.VAR & PLSUBMIT 。COM & PLSIN.FOR & RUNINF.FOR & $\begin{array}{l}\operatorname{READ}(\#, *) \text { NLOOP } \\
\operatorname{READ}(\#, *) \text { NFRUN }\end{array}$ & $\begin{array}{l}\text { A user-transparent, tert } \\
\text { porary file. }\end{array}$ \\
\hline
\end{tabular}


Table 4: File Description, Pulsed Model (continued)

\begin{tabular}{|c|c|c|c|c|c|}
\hline Name of File & $\begin{array}{l}\text { Program Which } \\
\text { Creates File } \\
\end{array}$ & $\begin{array}{l}\text { Opening } \\
\text { Routine }\end{array}$ & $\begin{array}{l}\text { Routines } \\
\text { Which } \\
\text { Read File } \\
\end{array}$ & Read Statements & Contents \\
\hline \multirow[t]{2}{*}{ fllename-RND } & PLSCW.COM & RDPLS.FOR & RDPLS.FOR & 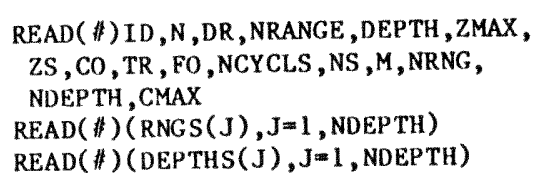 & $\begin{array}{l}\text { Values of the pressure } \\
\text { needed for the pulse } \\
\text { plots. }\end{array}$ \\
\hline & & & RDFREQ.FOR & $\begin{array}{c}\text { for } \begin{array}{c}I=1, N R N G \\
\operatorname{READ}(\#)(\operatorname{PSIR}(I, L), \operatorname{PSII}(I, L), \\
L=1, \operatorname{NDEPTH})\end{array}\end{array}$ & \\
\hline fillename.PLS & PLSFILE.COM & RDPLS . FOR & RDPLS . FOR & 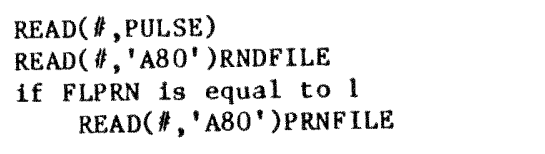 & $\begin{array}{l}\text { Specification information } \\
\text { for the pulse plots. }\end{array}$ \\
\hline filename.PRN & PULSE.EXE & PRNPRE.FOR & $-\infty$ & PRINT filename.PRN & $\begin{array}{l}\text { Values of the pressure as } \\
\text { a function of time for a } \\
\text { selected depth and range; } \\
\text { may be printed out by the } \\
\text { user. }\end{array}$ \\
\hline
\end{tabular}


\#, unit number used in the OPEN statement for a particular file.

*, Fortran symbol for free format.

AMP, real variable, amplitude of the temporal source function.

BOTFAC, real variable, factor used to generate the artificlal layer, i.e., BOTFAC*DEPTH $=$ ZMAX.

BOTTOM, namelist variable, NAMELIST/BOTTOM/DEPTH, BOTFAC.

Box, integer variable, flag indicating if a box are to be drawn around the contour plot.

CMAX, real variable, maximum sound speed amoung the sound speed profiles.

CONFILE, character variable, user-supplied file name for the file which contains values of the pressure needed for the contour plots; the file with the recommended extension .PRE.

CONTUR, namelist variable, NAMELIST/CONTUR/LOWLEV, LEVINV, NLEVEL, BOX, GRID, TICKS , FLABL , DEVICE , PLTRNG.

C0, real variable, reference sound speed (meters/second).

DEPFILE, character variable, user-supplied file name for the file which contains the values of the pressure needed for the line plots of transmission loss, i.e., the file with the recommended extension .DEP.

DEPTH, real variable, depth of the basin (meters). DEPTHS, real array, user-selected depths. DEVICE, integer variable, device number for graphics plots. DINDEX, integer 1-dimensional array, index values for the depths which bracket the depths selected for line plots. 
DPRES, real variable, interval between ticks on the y-axis of the plot of the pressure (meters).

DR, real variable, range step (meters).

DRANGE, real variable, interval size between ticks on the $x$-axis (k1lometers).

DTRLOS, real variable, interval size between ticks on the y-axis of the plot of transmission loss.

F, real varlable, acoustic frequency (Hertz).

F0, real varlable, carrier frequency (Hertz).

FLABL, integer variable, flag indicating if plot axes are to be labe1led.

FLMXMN, integer variable, flag indicating how the minimum and maximum values are to be selected for the plot.

FLPRN, integer variable, flag indicating if the values of the pressure are to be printed.

FLTICK, integer variable, flag indicating if ticks marks are to be drawn.

GRID, integer variable, flag indicating if a background grid is to be drawn.

ID, character variable, title for a particular run. LEVINV, real variable, increment between contour levels. LOWLEV, real variable, lowest contour level. $M$, integer variable, transform size is equal to $2 * * M$. MXTRLS, real variable, maximum transmission loss. MNTRLS, real variable, minimum transmission loss. MNPRES, real variable, minimum value of the pressure. MXPRES, real varlable, maximum value of the pressure. 
$\mathrm{N}$, integer variable, transform size is equal to $2 * *_{N}$.

NCYCLS, integer variable, number of cycles.

NDEPTH, integer variable, number of depths for which the pressure at selected ranges would be written out to file.

NFRUN, integer variable, number of frequencies for which the pressure is to be calculated in a particular run.

NLEVEL, integer variable, number of contour levels to be plotted; a flag indicating that the number of levels are to be determined by the program.

NLOOP, integer variable, indicates the frequencies for which the pressure has been calculated.

NPTS, integer variable, transform size $\left(2 * *_{N}\right)$.

NPTSVP, integer variable, number of sound speed values in each sound speed profile.

NRANGE, integer variable, number of ranges values in the computation. NRNG, integer variable, number of ranges for which the pressure is written out to disk.

NS, integer variable, number of samples per cycles.

NSVP, integer variable, number of sound speed profiles in the file.

OUTPUT, namelist variable, NAMELIST/OUTPUT/PLZINV, PLRINV, NDEPTH.

PHASE, integer variable, flag indicating if the phase is to be plotted.

PLRINV, integer variable, range increment for the transmission loss

plots is PLRINV*DR.

PLSFRE, namelist variable, NAMELIST/PLSFRE/TR, FO, NCYCLS, NS.

PLTRNG, real variable, range interval over which the transmission loss

is plotted (kilometers) or a flag indicating that the range interval is to be determined by the program. 
PLTZ, namelist variable, NAMELIST/PLTZ/FLMXMN,FLTICK, FLABL,DEVICE, MXTRLS , MNTRLS , DRANGE , DTRLOS , PLTRNG.

PLZINV, integer variable, depth increment for the transmission loss plots is PLZINV*DZ.

POUTPT, namelist variable, NAMELIST/POUTPT/NRNG, NDEPTH.

PRNFILE, character variable, user supplied file name for the file from which the values of the pressure as a function of time may be printed out, i.e., the file with the recommended extension .PRN.

PSII, real, 2-dimensional array, imaginary values of the pressure. PSIR, real, 2-dimensional array, real values of the pressure. PULSE, namelist variable, NAMELIST/PULSE/AMP, DEVICE, FLMXMN, TICKS, FLABL, PHASE, SECPLT , MXPRES , MNPRES , SECINV , DPRES , FLPRN.

RMAX, real variable, maximun range for the computation (kilometers). RNDFILE, character variable, user-supplied file name for the file which contains the values of the pressure needed for the pulsed plots, i.e., the file with the recommended extension .RND. RNGS, real, l-dimeinsional array, user-selected ranges. RNGSVP, real, 1-dimensional array, ranges corresponding to the profiles (kilometers).

SECINV, real variable, interval between tick marks on the x-axis of the plot of pressure verses time (seconds).

SECPLT, real variable, length of the x-axis of the plot of pressure vs. time (seconds).

SIZE, namelist variable, NAMELIST/SIZE/N, RMAX,DR. SOURCE, namelist variable, NAMELIST/SOURCE/ZS,F,CO. SVP, namelist variable, NAMEL IST/SVP/NSVP, NPTSVP. 
SVPFILE, character variable, user-supplied file name for the file which contains the values of the sound speed as a function of depth and range, $1 . e .$, the file with the recommended extension .SVP.

TICKS, Integer variable, flag indicating if ticks are to be drawn.

TR, real variable, repetition period (seconds).

VELSVP, real, 1-dimenstonal array, sound speed values for each profile (meters/second).

Z, real, 1-dimensional array, depth values for each profile (meters).

ZMAX, real variable, ocean depth plus thickness of the artificial layer (meters).

$\mathrm{ZS}$, real variable, source depth (meters).

75 
C. Utility Programs

The following DCL programs were created to compile, link or print the routines in the model:

\begin{tabular}{cccc}
\hline Mode1 Version & Compile & Link & Print \\
\hline CW & CPLCW.COM & LNKCW.COM & PRNCW.COM \\
CW contour's plot & CPLCON.COM & LNKCONIGL.COM & PRNCON.COM \\
CW line plot & CPLLPD.COM & LNKLPD.COM & PRNLPD.COM \\
Pulsed CW & CPLPCW.COM & LNKPCW.COM & PRNPCW.COM \\
Pulse and plot & CPLPLS.COM & LNKPLS.COM & PRNPLS.COM \\
DCL programs & & & PRNCOM.COM \\
\hline
\end{tabular}

The program that specifies all of the dimensions is [OAD.GONZALEZ. PPEMAT] PARAM.FOR. It specifies the maxinun number of locations in the arrays for both versions of the model. It is "included" in every program where arrays are dimensioned. To change the dimension of the arrays the user must

(1) change the appropiate parameter statement in PARAM.FOR,

(2) compile all the routines using the utility programs,

(3) 1ink the programs into executable modules using the utility programs. 


\section{SAMPLE RUNS}

Several sample runs were performed to demonstrate various features of the model. Figures 12 and 13 show output from the $\mathrm{CW}$ version produced with an artificial sound speed profile. These two figures illustrate the two different types of contour plots for the transmission loss values. Figure 12 is a contour using the UNIRAS plot routines and Figure 13 is the corresponding plot using the IGL color line contours. The sound speed profile for these plots is a parabolic function in depth, with the sound axis (sound speed minimum) occuring at $z=3000$ m. The total depth of the basin is $6000 \mathrm{~m}$. Hence the source is located mid-way in the water column at the sound axis. This environment produces a pattern of sound transmission where the ray paths are focused in a repetitive manner. When the ray paths are focused, i.e., in a convergence zone, a loud region exists. These conditions produce the rather symmetric pattern with respect to the mid-depth of the transmission loss values, and the existence of a loud region at a depth of $3000 \mathrm{~m}$ and a range of $60 \mathrm{~km}$. The differences in symmetry result frorn the difference in the treatment of the bottom of the ocean, and the sea surface. If the source depth is varied with a profile of this sort, the location of the convergence zone will shift in depth.

Figures 14, 15, and 16 were produced using a real sound speed profile from the Sargasso Sea. The sound speed axis for these data occurs at a depth of $1200 \mathrm{~m}$ and the bottom is at $5500 \mathrm{~m}$. These figures illustrate the differences in the convergence zone patterns that result from locating the source at different depths. The $\mathrm{CW}$ version was run with a frequency of $130 \mathrm{~Hz}$. The source depths are $130 \mathrm{~m}, 1200 \mathrm{~m}$, and $5200 \mathrm{~m}$ respectively. It is interesting to note the interaction of the 


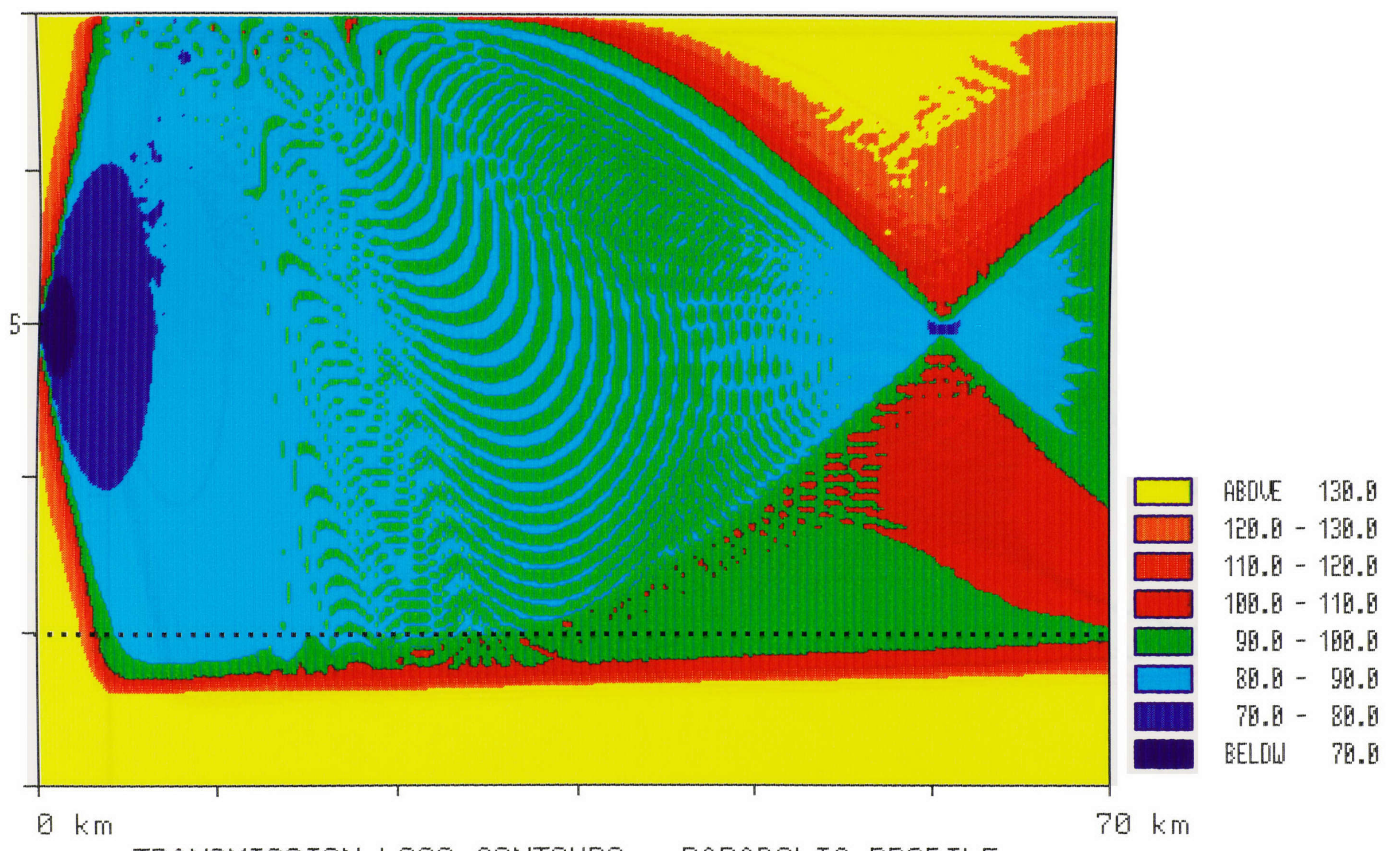

TRANGMISSION LOSS CONTOURS - FARABOLIC PROFILE EOTTOM DEPTH $=6000$ M SOURCE DEFTH $=3000 \mathrm{M} \quad \mathrm{FQ}=130 \mathrm{HZ}$ Figure 12. Transmission Loss Contours - Parabolic Profile (Uniras). 


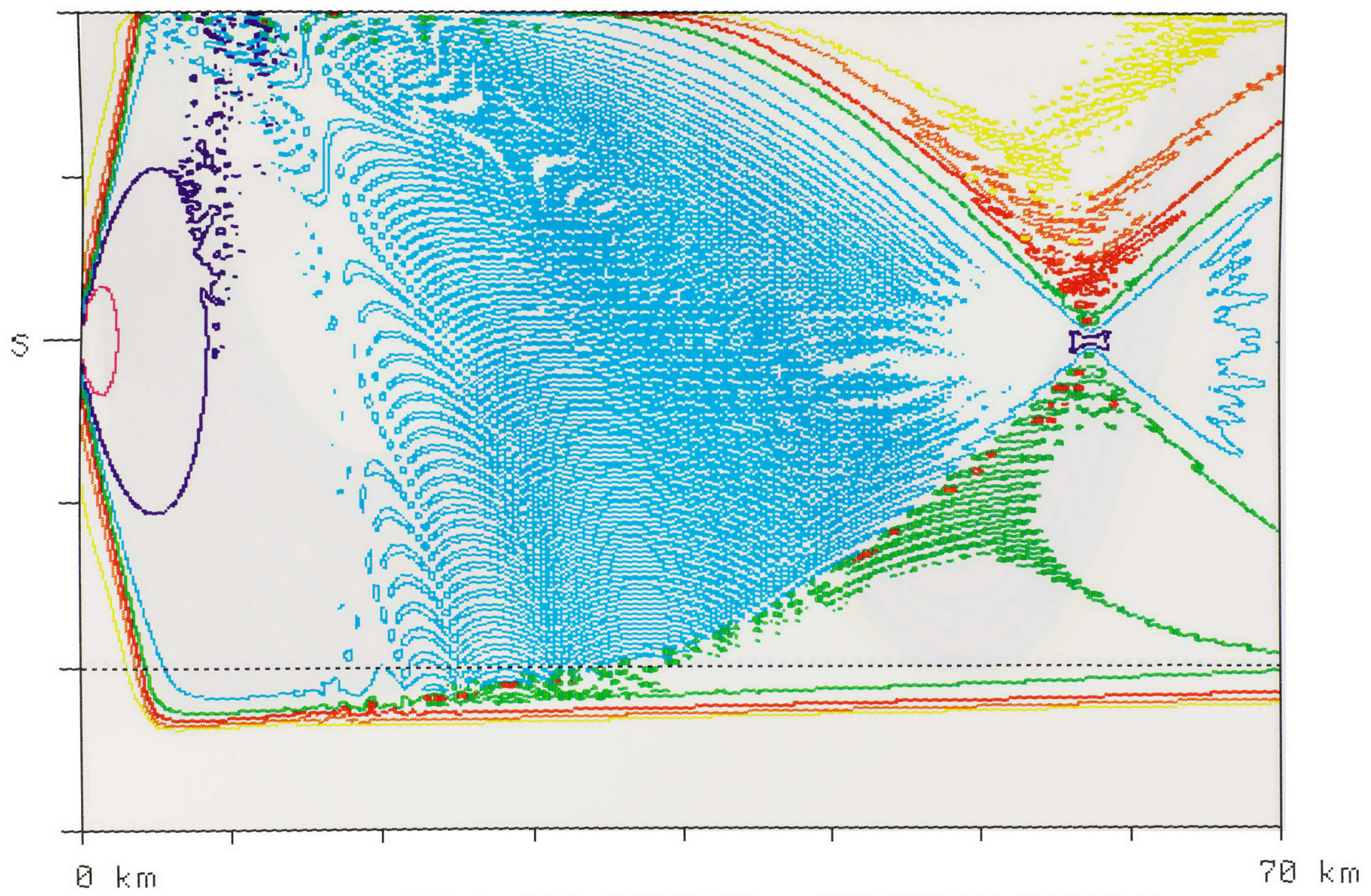

TRAYSMISSIDN LOSS CONTOLRS - FARAEDLIC PRDF ILE

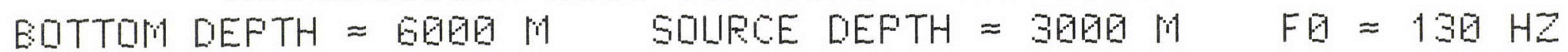




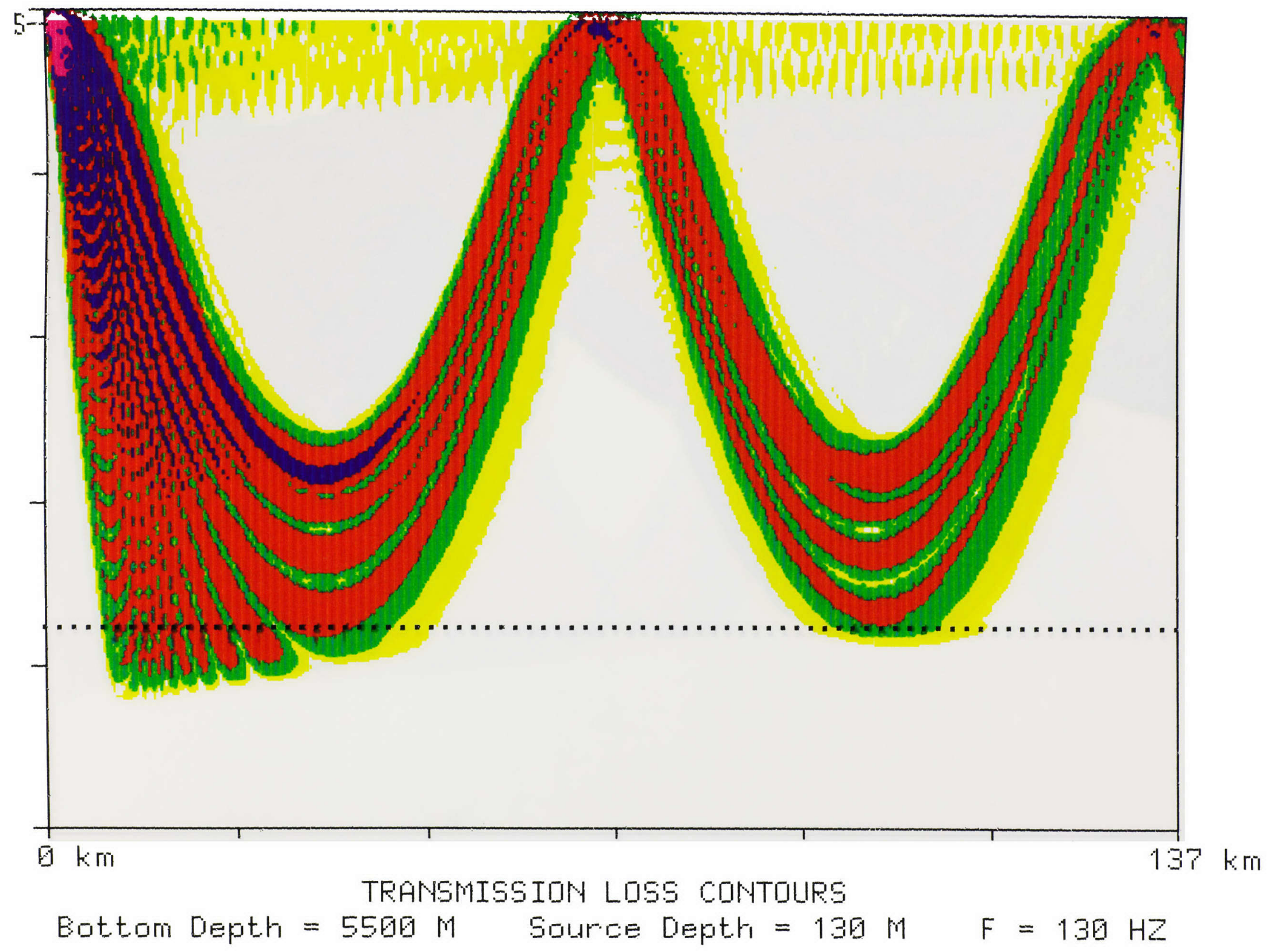

Figure 14. Transmission Loss Contours - Source Depth 130 Meters. 


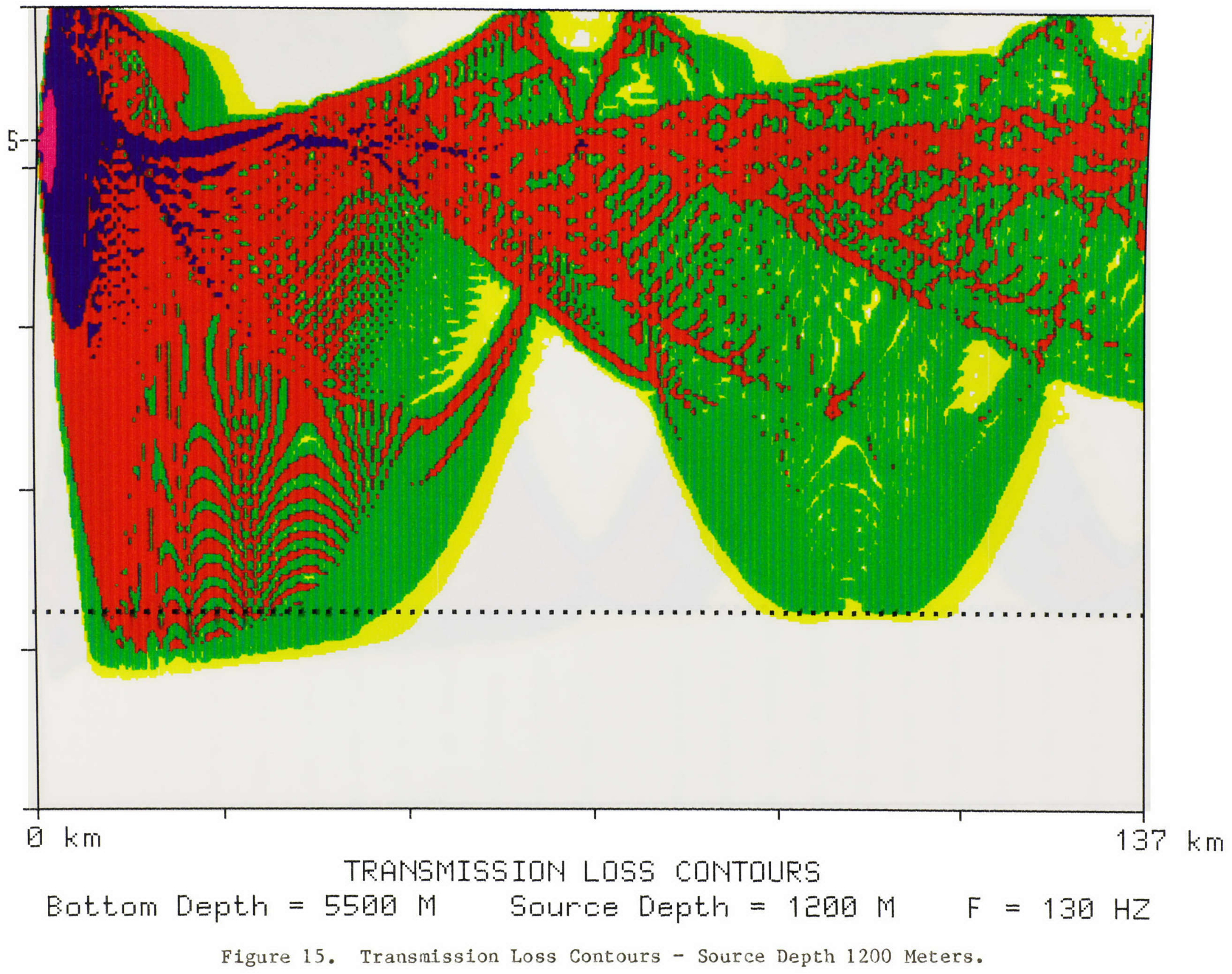




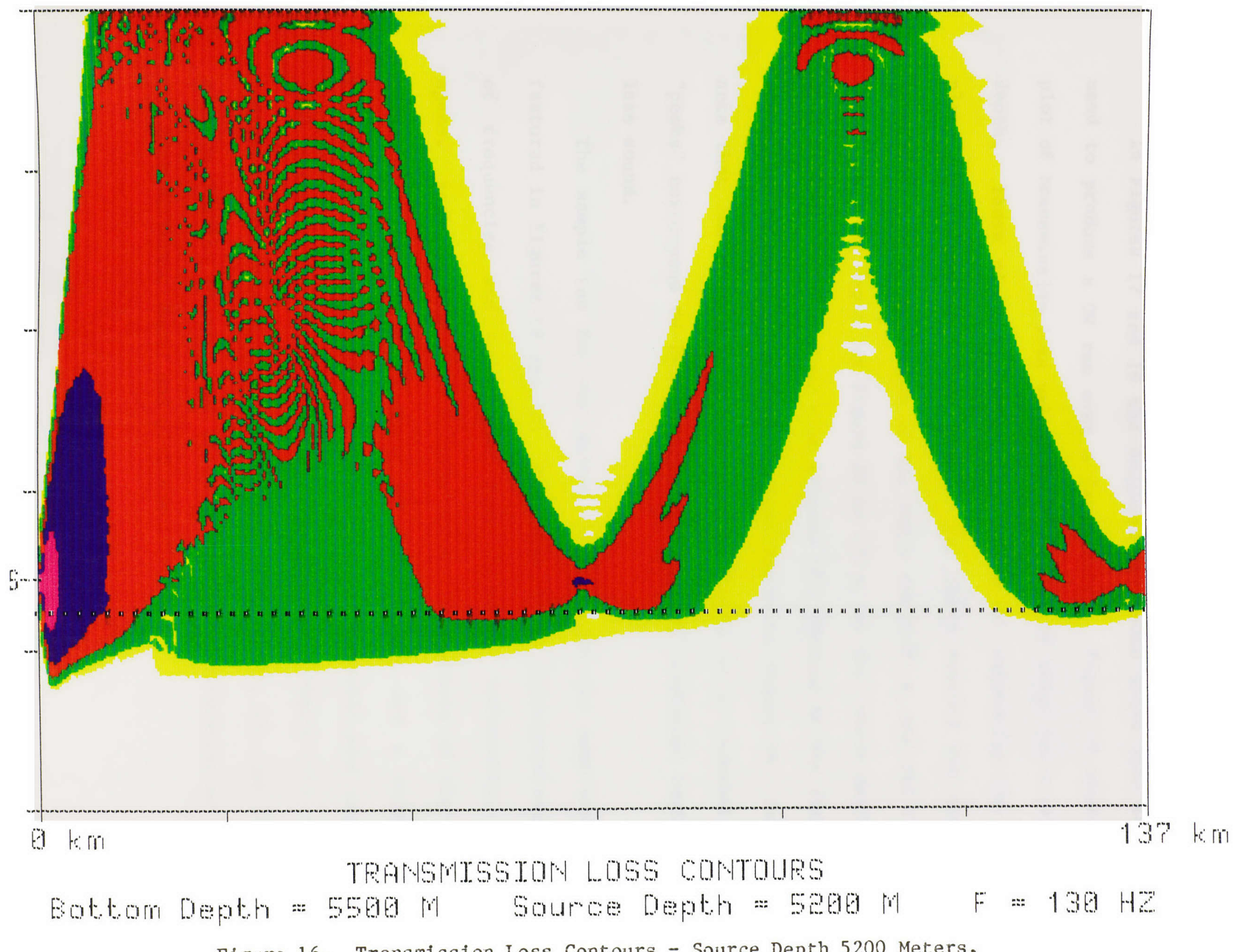

Figure 16. Transmission Loss Contours - Source Depth 5200 Meters. 
sound with the bottom in Figure 16, in contrast to the pattern obtained in Figure 15 when the source is located at the axis.

In Figures 17 and 18 the Sargasso Sea sound speed data was again used to produce a CW run over a large range. Figure 18 shows a line plot of transmission loss values as a function of range for a particular depth. These plots illustrate the method of segmenting the contour plots and transmission loss plots. For these results the source was located at $130 \mathrm{~m}$, the depth of the basin was $5500 \mathrm{~m}$, and the frequency was $50 \mathrm{~Hz}$. The depth for Figure 18 is $130 \mathrm{~m}$ (at the source depth). The loud regions in the contour plot (Figure 17), denoted by the red colors, correspond to the low transmission loss values in Figure 18. One should note that the transmission loss axis in Figure 18 is inverted so that "peaks" correspond to loud regions and "valleys" represent regions with less sound.

The sample run for the pulsed version used the same environment featured in Figures 12 and 13. The pressure was calculated over a band of frequencies and transformed from frequency information to time space. Approximately 39.5 seconds after the emission of the sound, a pulse was received at a depth of 3000 meters and a range of 60 kilometers (the loud region) (Figure 19). A comparable plot was done at 1000 meters (Figure 20) and at 5000 meters (Figure 21) in depth indicating arrivals at different times. Figures 22, 23, and 24 are the same as Figures 19,20 , and 21 correspondingly, illustrating the same run with different label and plot specifications. 


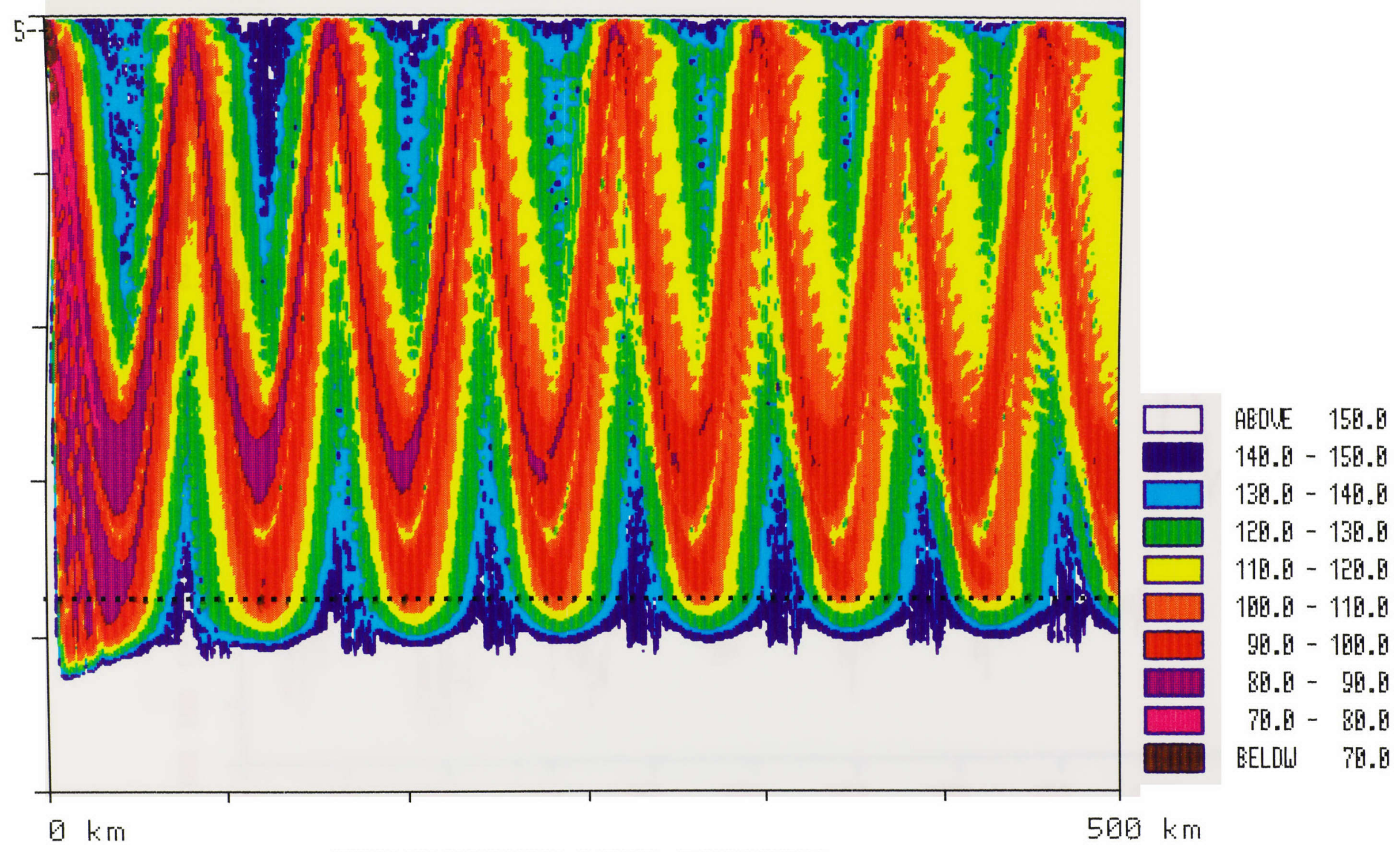

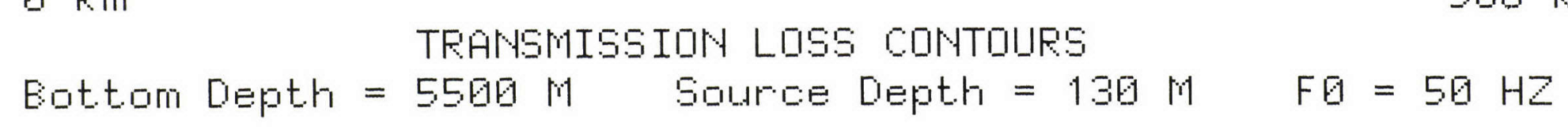

Figure 17. Transmission Loss Contours - Range 500 Kilometers. 


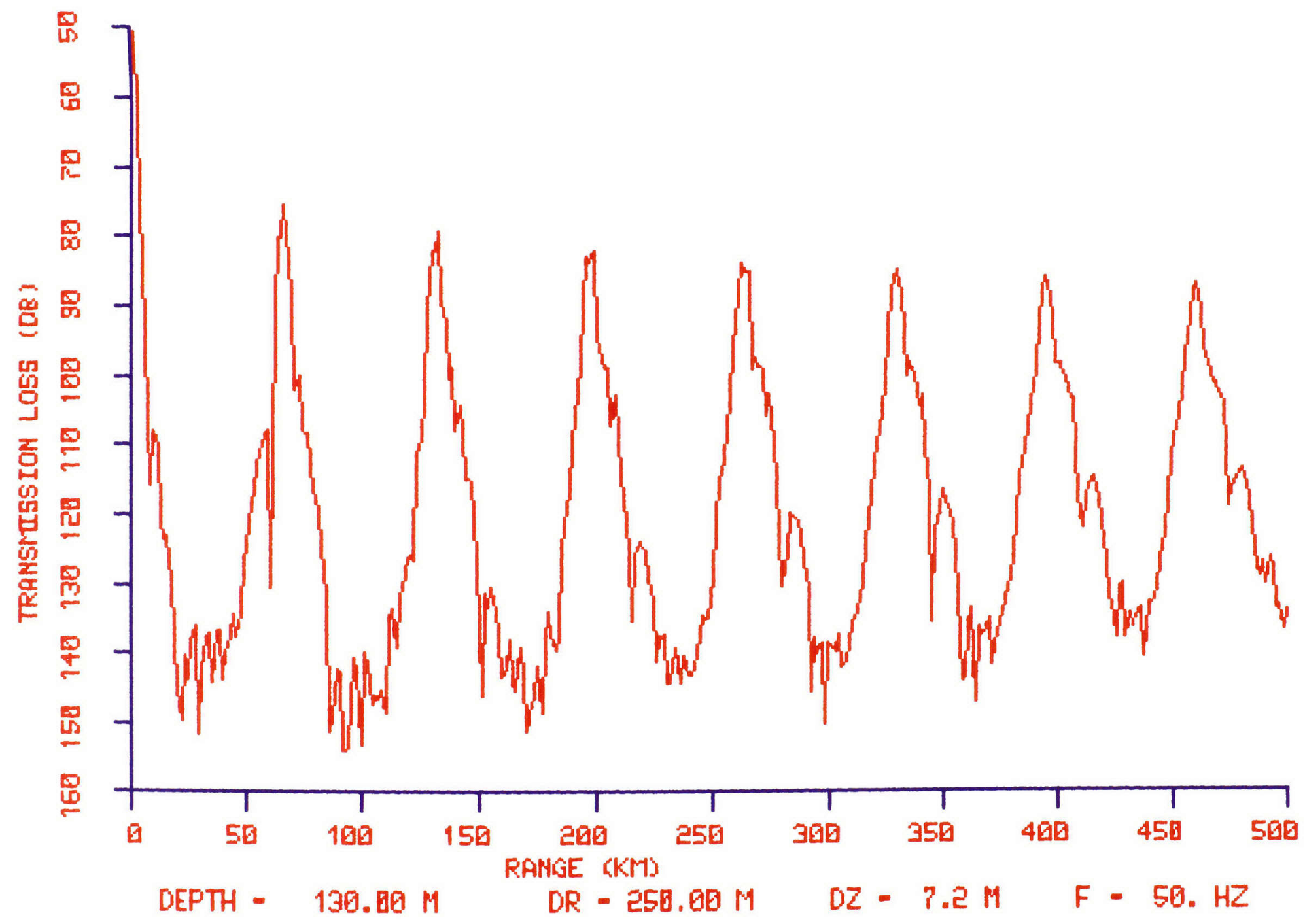

Figure 18. Line P1ot - Depth 130 Meters, Range 500 Kilometers. 


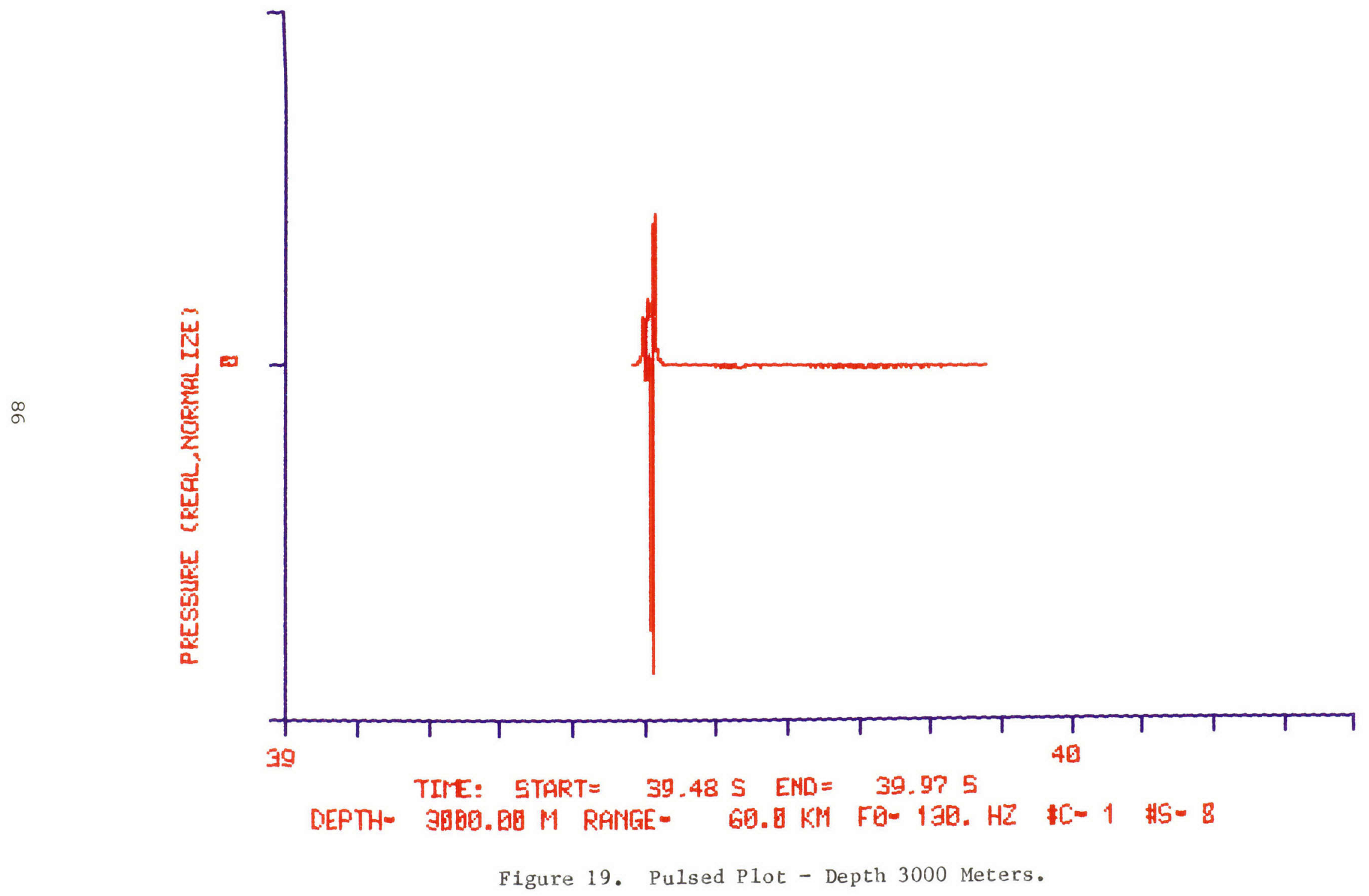




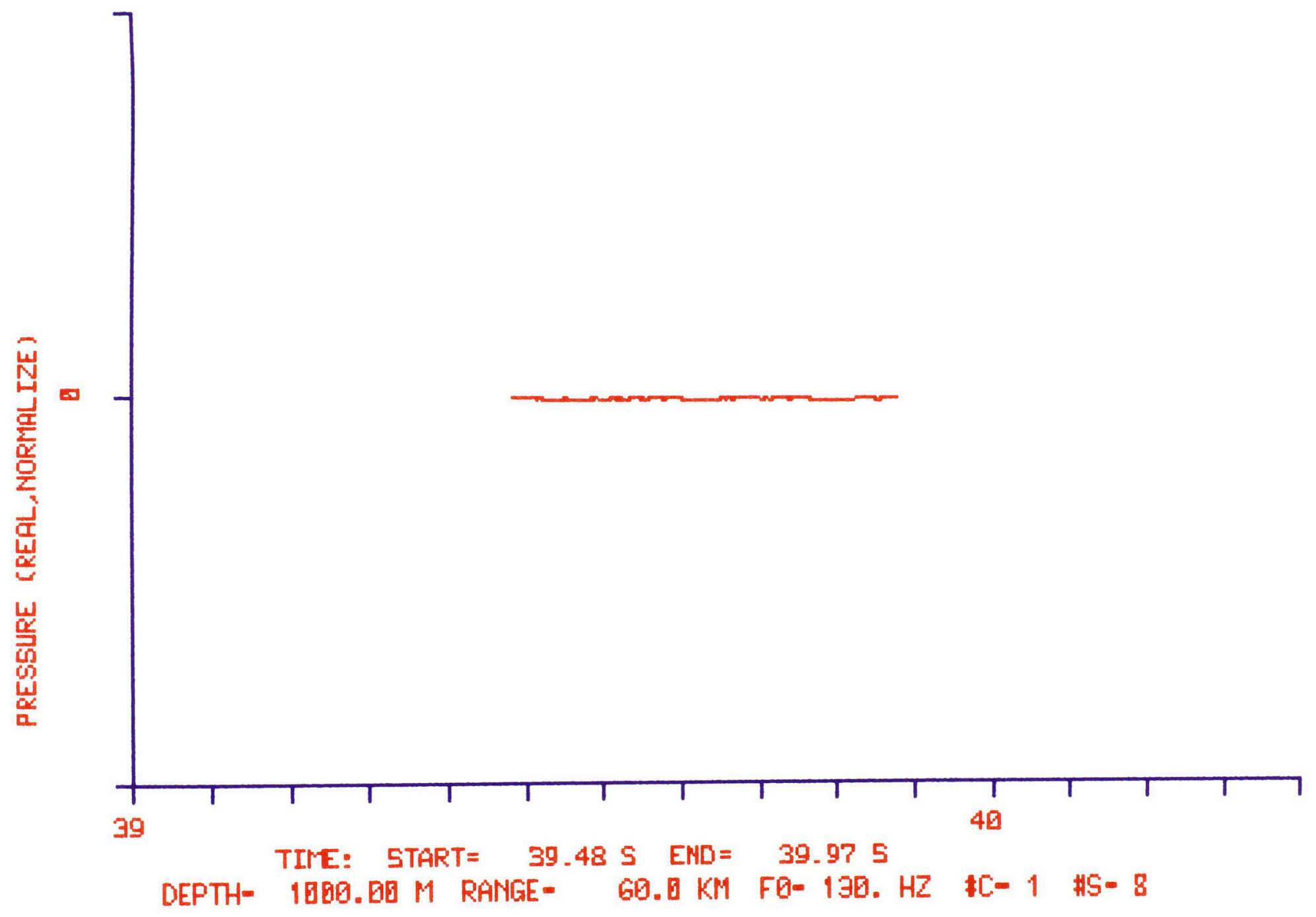

Figure 20. Pulsed Plot - Depth 1000 Meters. 


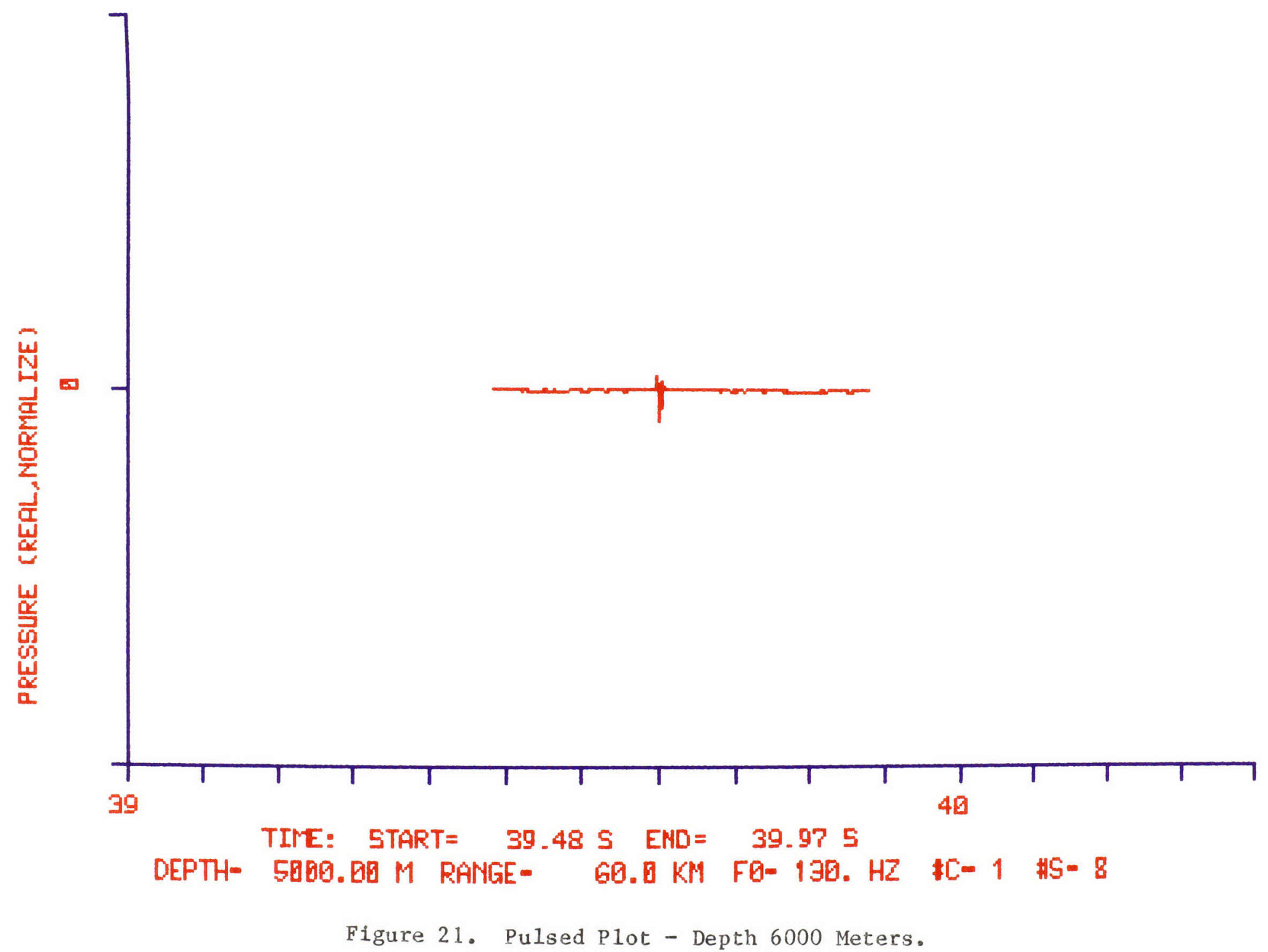




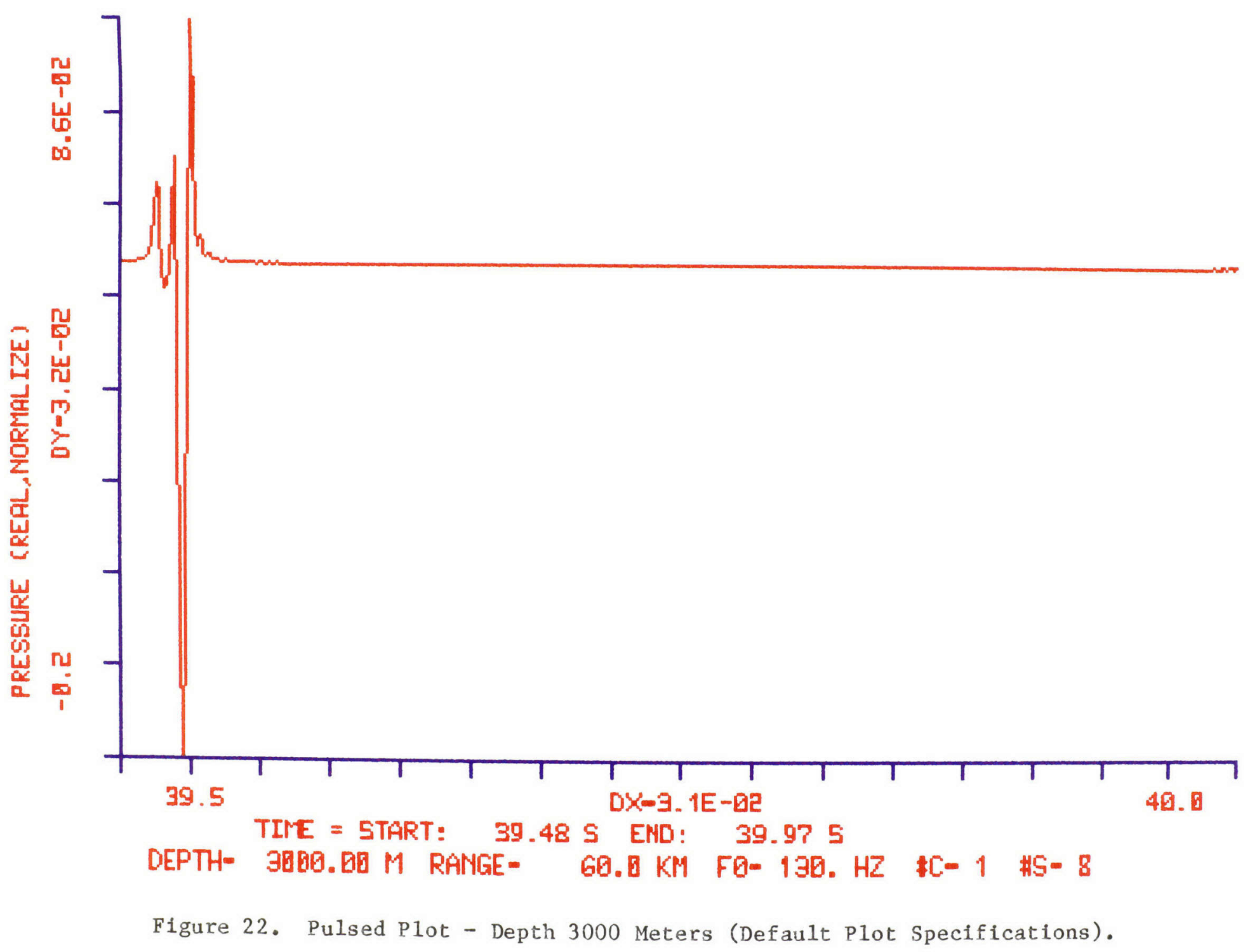




$$
\text { Hzw }
$$




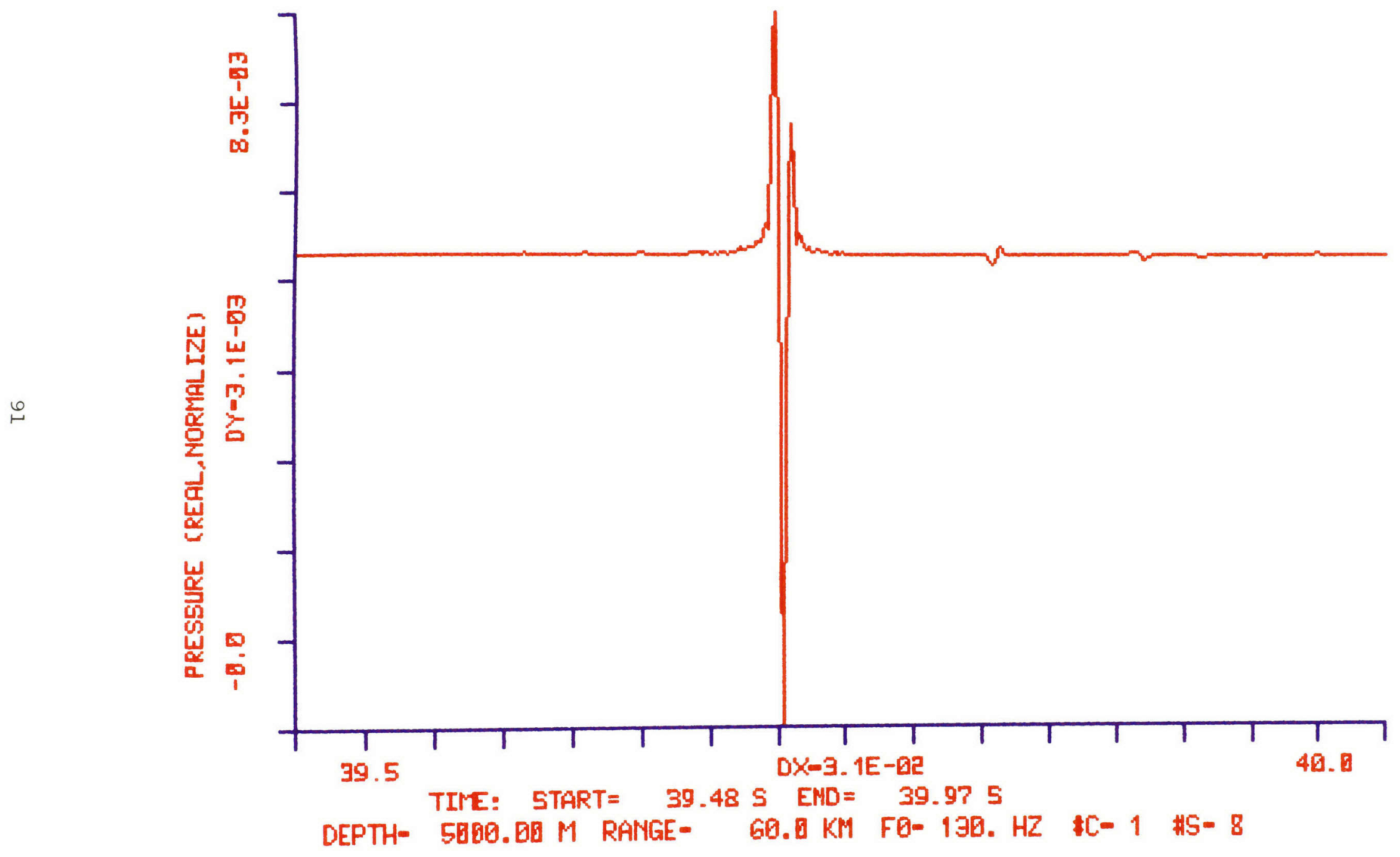

Figure 24. Pulsed Plot - Depth 6000 Meters (Default Plot Specifications). 
VII. FUTURE MODIFICATIONS

The modular design of the model makes it very flexible and expandable. It is not only user-friendly but also programmerfriendly. A11 the routines are structured and thoroughly documented. The "small subroutines" approach proved to be the best in order to make the model not only more understandable but easier to change and/or expand. For example, if a non-flat bottom was desired, all that would be required is:

(1) To write a subroutine that calculates the bottom depth given the range (this could be done using a function or a table) and, that given the bottom depth, would calculate the number of points in the water and the number of points in the artificial layer.

(2) This subroutine should be called before the calculation of the second coefficient $\left(c_{2} \cdot\right)$, i.e., in subroutine $c_{2}$, before CALL VELCTY. For detailed explanation of the structure of the model and its subroutine, see section III. MODEL DESCRIPTION.

To have several different bottoms all that is required is to have several routines like the one specified in number (1) and link them into several executables.

other things that could be done to improved the model are:

(1) Instead of using sequential access files, use direct access files. This would make the plotting of the transmission loss versus range for selected depths (CW version) more flexible and the plotting of the pulsed (PSP version) more efficient. 
(2) Use a variable name in references to programs in the DCL procedures. In the DCL procedures all the references to the program nodes or the scratch node are made using. the actual directory specification. It would be better if changes were made to use a variable name that is set to the actual directory at the beginning of the driver program. This would make it easier to copy the model from one node to another within the same computer or to copy it to another computer with DCL language facilities.

(3) The contour routines could be improved by offering more options for the labels, simflar to the line-plot package.

(4) The DCL command procedure that submits the batch jobs (PLSUBMIT.COM) could be improved to have a variable number of processors whose value is set inside the program. The program would then have the ability to submit and synchronize one or many jobs depending on the number of processors available by just making a trivial change. 
VIII. REFERENCES

1. G. Arfken, Mathematical Methods for Physicists (Academic Press, New York, 1970).

2. G. D. Bergland, "A Radix-Eight Fast Fourler Transform Subroutine for Rea1-Valued Series," IEEE Trans. Audio Electroacoust. AU-17, 138-144 (1969).

3. E. Oran Brigham, The Fast Fourier Transform (Prentice-Hall, Englewood Cliffs, New Jersey, 1974).

4. H. K. Brock, "The AESD Parabolic Equation Model," Naval Ocean Research and Development Activity Tech. Note 12 (1978).

5. C. S. Clay and H. Medwin, Acoustical Oceanography: Princtples and Applications (John Wiley and Sons, New York, 1977).

6. J. W. Cooley and J. W. Tukey, "An Algorithm for the Machine Calculation of Complex Fourier Series," Math. Comput. 19, 297 (1965).

7. J. W. Cooley, P. A. W. Lewis, and P. D. Welch, "The Fast Fourier Transform Algorithm: Programing Consideration in the Calculation of Sine, Cosine, and Laplace Transform," Journal of Sound and Vibration $12,315-337$ (1970).

8. J. A. Davis and R. C. Cavanagh, "NORDA Parabolic Equation Workshop," Naval Ocean Research and Development Activity Technical Note $143(1981)$.

9. R. H. Hardin and F. D. Tappert, "Applications of the Split-Step Fourier Method to the Numerical Solution of Nonlinear and Variable Coefficient Wave Equations," SIAM Rev. 15, 423 (1973).

10. R. J. Higgins, "Fast Fourier Transform: An Introduction With Some Minicomputer Experiments," Am. J. of Phys. 44, 766-773 (1976). 
11. F. Jensen and $\mathrm{H}$. Krol, "The Use of the Parabolic Equation Method in Sound Propagation Modelling," SACLANTCEN Memo SM-72 (1975).

12. M. J. Lighthi11, Introduction to Fourier Analysis and Generalized Functions (Cambridge Press, Cambridge, 1964).

13. S. T. McDaniel, "Application of the Parabolic Approximation to Predict Acoustical Propagation in the Ocean," Am. J. of Phys. 47, 63-68 (1979).

14. "Sound in the Ocean," Oceanus 20 (Spring, 1977).

15. C. W. Spofford, "A Synopsis of the AESD Workshop on Acoustic Propagation Modelling by Non-Ray Techniques," Acoustic Environmental Support Detachment Tech. Note TN-73-05 (1973).

16. F. D. Tappert, in Lecture Notes in Physics, vol. 70, Wave Propagation and Underwater Acoustics, edited by J. B. Keller and J. S. Papadakis (Springer-Verlag, Berlin, 1977).

17. F. D. Tappert and R. H. Hardin, in "A Synops is of the AESD Workshop on Acoustic Modeling by Non-Ray Tracing Techniques," Acoustic Environmental Support Detachment Tech. Note TN-73-05 (Nov. 1973).

18. F. D. Tappert, "Parabolic Equation Method in Underwater Acoustics," J. Acoust. Soc. Am. 55, S34(A) (1974). 
IX. VITA

Title of Thesis: PULSED PARABOLIC EQUATION MODEL OF ACOUSTIC TRANSMISSION

Name of Candidate: Allcia Gonzalez Acebo

Place and Date of Birth: La Habana, Cuba; March 25, 1948

Citizenship: U.S.A.

Colleges and Universities:

Universidad de Puerto Rico, Rio Piedras, PR (1965-1968)

B.A., Mathematics (graduated Magna Cum Laude)

Miami Dade Community College, Miami, FL (1976)

(12 undergraduate credit hours in computer science)

Florida International University, Miami, F1 (1976-Present)

(21 undergraduate and 21 graduate credit hours in computer science)

Professional Organizations:

Association for Computing Machinery

Publication:

"A Method for Automating the Construction of Irregular Computational Grids for Storm Surge Forecast Models," J. Comput. Phys., 37, 371-387 (1980) (with W. C. Thacker and G. E. Putland).

Major Department: Mathematical Science

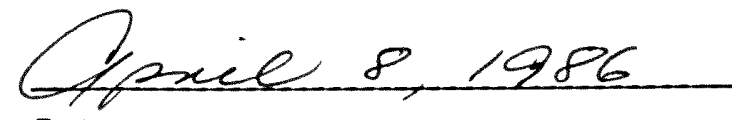

Date

Signed

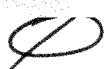

